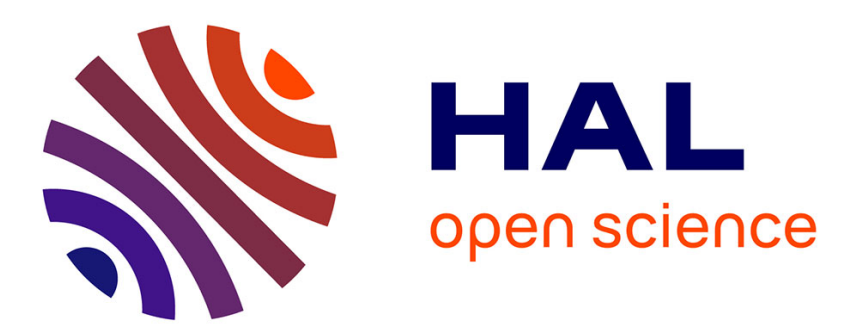

\title{
A technique of flux reconstruction at the interfaces of nonconforming grids for aeroacoustic simulations
}

\author{
Sophie Le Bras, Hugues Deniau, Christophe Bogey
}

\section{To cite this version:}

Sophie Le Bras, Hugues Deniau, Christophe Bogey. A technique of flux reconstruction at the interfaces of nonconforming grids for aeroacoustic simulations. International Journal for Numerical Methods in Fluids, 2019, 91 (12), pp.587-614. 10.1002/fld.4767 . hal-02351079

\author{
HAL Id: hal-02351079 \\ https://hal.science/hal-02351079
}

Submitted on 24 Nov 2020

HAL is a multi-disciplinary open access archive for the deposit and dissemination of scientific research documents, whether they are published or not. The documents may come from teaching and research institutions in France or abroad, or from public or private research centers.
L'archive ouverte pluridisciplinaire HAL, est destinée au dépôt et à la diffusion de documents scientifiques de niveau recherche, publiés ou non, émanant des établissements d'enseignement et de recherche français ou étrangers, des laboratoires publics ou privés. 


\title{
A technique of flux reconstruction at the interfaces of non-conforming grids for aeroacoustic simulations
}

\author{
S. Le Bras ${ }^{12 *}$, H. Deniau ${ }^{3}$ and C. Bogey ${ }^{4}$ \\ ${ }^{1}$ Centre Européen de Recherche et de Formation Avancée en Calcul Scientifique, 31057 Toulouse, France. \\ ${ }^{2}$ Siemens Industry Software N.V., 3001 Leuven, Belgium. \\ ${ }^{3}$ ONERA, French Aerospace Lab, 31000 Toulouse, France. \\ ${ }^{4}$ Univ Lyon, Ecole Centrale de Lyon, INSA Lyon, Université Claude Bernard Lyon I, CNRS, Laboratoire de Mécanique \\ des Fluides et d'Acoustique, UMR 5509, F-69134, Ecully, France.
}

\section{SUMMARY}

A flux reconstruction technique is presented in order to perform aeroacoustic computations using implicit high-order spatial schemes on multiblock structured grids with non-conforming interfaces. The use of such grids, with mesh spacing discontinuities across the block interfaces, eases local mesh refinements, simplifies the mesh generation process, and thus facilitates the computation of turbulent flows. In this work, the spatial discretization consists of sixth-order finite-volume implicit schemes with low-dispersion and lowdissipation properties. The flux reconstruction is based on the combination of non-centered schemes with local interpolations to define ghost cells and compute flux values at the grid interfaces. The flow variables in the ghost cells are calculated from the flow field in the grid cells using a meshless interpolation with radial basis functions. In this study, the flux reconstruction is applied to both plane and curved non-conforming interfaces. The performance of the method is first evaluated by performing two-dimensional simulations of the propagation of an acoustic pulse and of the convection of a vortex on Cartesian and wavy grids. No significant spurious noise is produced at the grid interfaces. The applicability of the flux reconstruction to a 3-D computation is then demonstrated by simulating a jet at a Mach number of 0.9 and a diameter-based Reynolds number of $4 \times 10^{5}$ on a Cartesian grid. The non-conforming grid interface located downstream of the jet potential core does not appreciably affect the flow development and the jet sound field, while reducing the number of mesh points by a factor of approximately two.

Copyright (c) 2018 John Wiley \& Sons, Ltd.

KEY WORDS: non-conforming grids, structured grids, high-order schemes, finite volumes, meshless interpolation, aeroacoustics

\section{INTRODUCTION}

12 For flows at high Reynolds numbers, the direct computation of the aerodynamic noise from the Navier-Stokes equations requires accurate numerical methods in order to properly compute both the

\footnotetext{
${ }^{*}$ Correspondence to: Sophie Le Bras, Siemens Industry Software N.V., Interleuvenlaan 68, 3001 Leuven, Belgium.

E-mail: sophie.le_bras@siemens.com 
small turbulent motions and the low-frequency sound waves in the radiated pressure field [1-3]. In order to meet these requirements, in addition to high-order discretization schemes, locally refined meshes are needed in order to capture the turbulent eddies generating noise [4].

For aeroacoustic simulations performed on multiblock structured grids, the computational domain is usually divided into subdomains composed of conforming grids characterized by a full pointmatching distribution at the block interface, as shown in Fig. 1(a). Difficulties in performing highfidelity computations with such grids arise when the geometries are complex. Such geometries must be included in the numerical simulations in order to faithfully reproduce the conditions of the experiments [5,6]. In this context, high-quality structured meshes with conforming interfaces are in many cases almost impossible to generate [7]. For instance, for high-speed flows exhausting from turbofan jet engines or developing on aircraft wings [8], extremely fine grids are required to resolve the flow in the boundary layers and the wakes. Using conforming grids, local mesh refinements can be found in all the computational domain, leading to an excessive number of mesh points as well as to the generation of extremely small cells in out of interest areas. Obviously, this increases the computational cost of the simulation. In addition, using an explicit time discretization scheme, the presence of very small mesh cells imposes severe constraints on the time step so that the CFL restriction is verified [3].

(a)

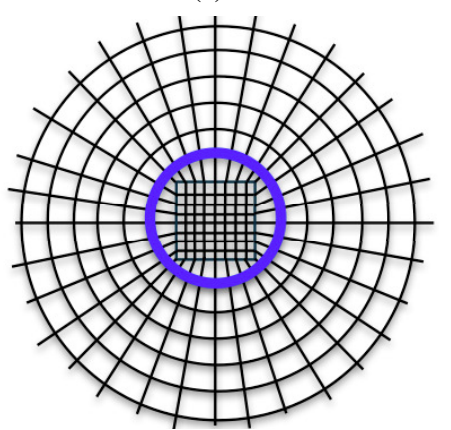

(b)

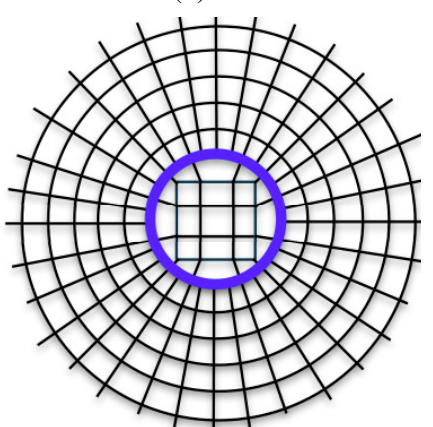

Figure 1. Representation of 2-D meshes with (a) conforming and (b) non-conforming grid interfaces in blue.

In order to perform aeroacoustic simulations of high-Reynolds-number flows at a reasonable computational cost, the use of non-conforming grids [9] without overlapping is attractive. Such meshes exhibit discontinuities of the grid lines across the block interface. This is the case of Fig. 1(b), providing an example of a non-conforming mesh with discontinuous grid spacings in the azimuthal direction at the block interface in blue. Using such a mesh for instance, the refinement at the center of the grid in Fig. 1(a) can be avoided. The size of the smallest cells and thus the time step are therefore chosen such that the acoustic sources are well-discretized. In addition, the use of non-conforming grids simplifies the grid generation process since the mesh blocks composing the computational domain can be created independently and then easily assembled. In return, in order to obtain high-fidelity numerical results using non-conforming grids, an accurate spatial discretization at the grid interfaces is required. Indeed, as the grid spacing is discontinuous at the block interface, the spatial discretization schemes cannot usually be applied close to the interface and their formulations have to be modified.

In computational aeroacoustics, the spatial discretization can be carried out using high-order low-dissipation and low-dispersion schemes, amongst which the dispersion-relation-preserving 
schemes [10], the optimized explicit schemes in the Fourier space [2], or the implicit schemes [11, 12]. In this study, the spatial discretization consists of the sixth-order finite-volume implicit scheme of Fosso et al. [12] in combination with the sixth-order implicit selective filter of Visbal and Gaitonde [13]. Implicit schemes are particularly attractive in order to reach a high-order spectral accuracy using a smaller number of grid points compared to explicit schemes. However, in the context of parallel computations, the flow equations are generally solved locally in each subdomain of the multiblock grid. As a consequence, the implicit centered schemes cannot be applied at the mesh block interfaces. Therefore, in a previous study [12], a technique of flux reconstruction at the interface of conforming grids has been developed. Based on the application of non-centered spatial schemes at the block interface and the use of ghost cells, the technique allowed us to successfully perform massively parallel aerodynamic and aeroacoustic computations of jet flows [14-17].

In the present study, a flux reconstruction technique for the interface of non-conforming grids is proposed. The technique, derived from the method developed for conforming grids [12], is based on the application of non-centered schemes at the grid interface. Due to the mesh line discontinuities at the grid interface, an additional step consisting in reconstructing ghost cells is required. The flow variables in the ghost cells are computed using a local interpolation technique, based on a meshless method involving Radial Basis Functions (RBF) [9, 18]. Meshless interpolations are useful in order to alleviate the difficulties caused by the loss of the mesh topology at the interfaces of non-conforming grids. Indeed, since meshless interpolations are performed from arbitrarily scattered spatial data without any geometrical information, computational overheads due to topology reconstructions are avoided. Originally developed by the authors for plane nonconforming grid interfaces [19], the technique of flux reconstruction is extended to curved interfaces in this study. In comparison with the preliminary results presented in [19], the properties of the RBF interpolation are examined in 1-D in the wavenumber space, and the performance of the flux reconstruction is further assessed by simulating in 2-D the convection of a vortex on wavy grids and the propagation of an acoustic pulse. In addition, the application of the technique to a threedimensional turbulent jet flow is presented.

The present paper is organized as follows. In a first section, the high-order finite-volume approach used in this study and the flux reconstruction method for conforming interfaces are described. In section 2, the reconstruction technique developed at the interface of plane and curved nonconforming grids is presented. In section 3, the properties of the RBF interpolations are examined in 1-D in the wavenumber space. In section 4, the accuracy of the flux reconstruction is evaluated by simulating a two-dimensional acoustic pulse propagating through a non-conforming interface. In section 5, the simulations of two-dimensional vortex convection for Cartesian and wavy grids with different spatial resolutions are presented. In particular, the advantages of using RBF interpolations for the reconstruction and the choice of the interpolation parameters are discussed. Finally, the application of the technique to a 3-D turbulent jet flow is presented, using a non-conforming grid downstream of the jet potential core. The reduction in the number of mesh points obtained using a non-conforming grid is evaluated. The effects of the presence of a non-conforming interface on the sound field radiated by the jet are examined. 


\section{FLUX RECONSTRUCTION TECHNIQUE FOR CONFORMING GRIDS}

\subsection{Governing equations}

In this study, the 3-D compressible Navier-Stokes equations are solved. Using Cartesian coordinates, they can be written as:

$$
\frac{\partial \mathbf{W}}{\partial t}+\frac{\partial \mathbf{E}_{c}}{\partial x}+\frac{\partial \mathbf{F}_{c}}{\partial y}+\frac{\partial \mathbf{G}_{c}}{\partial z}-\frac{\partial \mathbf{E}_{d}}{\partial x}-\frac{\partial \mathbf{F}_{d}}{\partial y}-\frac{\partial \mathbf{G}_{d}}{\partial z}=0
$$

where $\left(\mathbf{E}_{c}, \mathbf{F}_{c}, \mathbf{G}_{c}\right)$ are the convective fluxes, $\left(\mathbf{E}_{d}, \mathbf{F}_{d}, \mathbf{G}_{d}\right)$ are the diffusive fluxes, $\mathbf{W}=(\rho, \rho u, \rho v, \rho w, \rho e)^{t}$ is the vector of the conservative variables, $\rho$ is the density, $(u, v, w)$ are the velocity components, and $\rho e$ is the total energy. For a perfect gas, the total energy $\rho e$ is given by:

$$
\rho e=\frac{p}{\gamma-1}+\frac{1}{2} \rho\left(u^{2}+v^{2}+w^{2}\right)
$$

where $p$ is the static pressure and $\gamma$ is the specific heat ratio. The convective fluxes write as:

$$
\left\{\begin{array}{l}
\mathbf{E}_{c}=\left(\rho u, \rho u^{2}+p, \rho u v, \rho u w,(\rho e+p) u\right)^{t} \\
\mathbf{F}_{c}=\left(\rho v, \rho u v, \rho v^{2}+p, \rho v w,(\rho e+p) v\right)^{t} \\
\mathbf{G}_{c}=\left(\rho w, \rho u w, \rho v w, \rho w^{2}+p,(\rho e+p) w\right)^{t}
\end{array}\right.
$$

and the diffusive fluxes as:

$$
\left\{\begin{array}{l}
\mathbf{E}_{d}=\left(0, \tau_{11}, \tau_{12}, \tau_{13}, \tau_{11} u+\tau_{12} v+\tau_{13} w+H_{1}\right)^{t} \\
\mathbf{F}_{d}=\left(0, \tau_{21}, \tau_{22}, \tau_{23}, \tau_{21} u+\tau_{22} v+\tau_{23} w+H_{2}\right)^{t} \\
\mathbf{G}_{d}=\left(0, \tau_{31}, \tau_{32}, \tau_{33}, \tau_{31} u+\tau_{32} v+\tau_{33} w+H_{3}\right)^{t}
\end{array}\right.
$$

where $\mathbf{H}=\left(H_{1}, H_{2}, H_{3}\right)^{t}$ is the heat flux vector, $\tau_{i j}=2 \mu S_{i j}$ is the viscous stress tensor, $\mu$ is the dynamic molecular viscosity computed from Sutherland's law, and $S_{i j}$ is the deformation stress tensor:

$$
S_{i j}=\frac{1}{2}\left(\frac{\partial u_{i}}{\partial x_{j}}+\frac{\partial u_{j}}{\partial x_{i}}-\frac{2}{3} \frac{\partial u_{k}}{\partial x_{k}} \delta_{i, j}\right)
$$

The heat flux vector $\mathbf{H}$ is computed from Fourier's law, yielding:

$$
\mathbf{H}=-\lambda \nabla T
$$

where $\nabla T$ is the temperature gradient, $\lambda=C_{p} \mu / \operatorname{Pr}$ is the thermal conductivity, $C_{p}$ is the specific heat at constant pressure, and $\operatorname{Pr}$ is the Prandtl number.

\subsection{High-order finite-volume approach}

The computations are performed using the finite-volume multi-block structured solver $\boldsymbol{e l s} \boldsymbol{A}$ [20], allowing us to perform Direct Numerical Simulations (DNS) or Large-Eddy Simulations (LES). In a finite-volume approach, the integral form of the Navier-Stokes equation (1) is solved at a discrete level. For this purpose, the computational domain is divided into non-overlapping control volumes $\Omega_{i}$, where $i$ is the volume index. Integrating Eq. (1) over the elementary volumes $\Omega_{i}$ and using the 
divergence theorem lead to:

$$
\left|\Omega_{i}\right| \frac{d \mathbf{U}_{i}}{d t}+\int_{\partial \Omega_{i}}\left(\mathbf{E}_{c}+\mathbf{F}_{c}+\mathbf{G}_{c}\right) \cdot \boldsymbol{n} \mathrm{d} S+\int_{\partial \Omega_{i}}\left(\mathbf{E}_{d}+\mathbf{F}_{d}+\mathbf{G}_{d}\right) \cdot \boldsymbol{n} \mathrm{d} S=0
$$

where $\boldsymbol{n}=\left(n_{x}, n_{y}, n_{z}\right)$ is the outgoing unitary normal of $\Omega_{i}, \partial \Omega_{i}$ represents the faces of $\Omega_{i}$ and $\mathbf{U}_{i}$ is the mean value of $\mathbf{W}$ in the volume $\Omega_{i}$ such as:

$$
\mathbf{U}_{i}=\frac{1}{\left|\Omega_{i}\right|} \int_{\Omega_{i}} \mathbf{W} \mathrm{d} V
$$

In the $\boldsymbol{e l} \boldsymbol{s} \boldsymbol{A}$ solver, the diffusive fluxes in Eq. (7) are calculated from the gradient $\nabla \mathbf{U}$ estimated at the cell interfaces using a 2nd-order method [21]. For clarity, in the following, only the convective fluxes are presented in the equations. Following Fosso et al. [12] and supposing that the volume $\Omega_{i}$ is an hexahedron, the normal $n$ is constant along the interface, and the integral of the convective fluxes in Eq. (7) can be approximated as:

$$
\int_{\partial \Omega_{i}}\left(\mathbf{E}_{c}+\mathbf{F}_{c}+\mathbf{G}_{c}\right) \cdot \boldsymbol{n} \mathrm{d} S \simeq\left|\partial \Omega_{i}\right|\left(\mathbf{E}_{c}\left(\widetilde{\mathbf{U}}_{\partial \Omega_{i}}\right) n_{x}+\mathbf{F}_{c}\left(\widetilde{\mathbf{U}}_{\partial \Omega_{i}}\right) n_{y}+\mathbf{G}_{c}\left(\widetilde{\mathbf{U}}_{\partial \Omega_{i}}\right) n_{z}\right)
$$

where $\widetilde{\mathbf{U}}_{\partial \Omega_{i}}$ is the averaged value of the variable vector $\mathbf{W}$ at the cell interface $\partial \Omega_{i}$ :

$$
\widetilde{\mathbf{U}}_{\partial \Omega_{i}}=\frac{1}{\left|\partial \Omega_{i}\right|} \int_{\partial \Omega_{i}} \mathbf{W} \mathrm{d} S
$$

The convective fluxes are thus computed from the interface-averaged values $\widetilde{\mathbf{U}}$ of the flow variables. In order to obtain a high-order calculation of the convective fluxes derivatives, a high-order interpolation of vector $\widetilde{\mathbf{U}}$ is performed from the cell-averaged values $\mathbf{U}$. Considering the onedimensional computational domain of Fig. 2, the interpolated vector $\widetilde{\mathbf{U}}$ at the interface $i+1 / 2$ is obtained by solving the implicit scheme:

$$
\alpha_{i+1 / 2} \widetilde{\mathbf{U}}_{i-1 / 2}+\widetilde{\mathbf{U}}_{i+1 / 2}+\beta_{i+1 / 2} \widetilde{\mathbf{U}}_{i+3 / 2}=\sum_{l=-1}^{2} a_{l} \mathbf{U}_{i+l}
$$

where $\alpha_{i+1 / 2}, \beta_{i+1 / 2}$ and $a_{l}$ are the scheme coefficients which are obtained from a 5th-order Taylor series [12]. This scheme correctly resolves the wavelengths discretized by at least 5 points [14]. Note that despite the use of approximation (9) which is formally only second-order accurate, Fosso et al. [12] demonstrated that the numerical scheme (11) is equivalent to Lele's 6th-order finitedifference scheme [11] for a uniform Cartesian mesh.

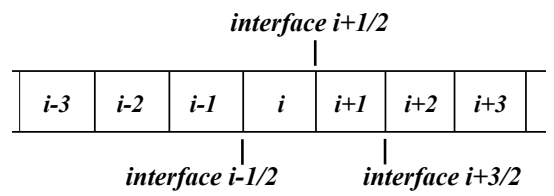

Figure 2. Representation of a one-dimensional computational domain.

In order to ensure the stability of the centered scheme (11), the 6th-order compact filter of Visbal and Gaitonde [13] is applied to the flow variables. The filtered values, denoted $\widehat{\mathbf{U}}$, are estimated 
from the values of $\mathbf{U}$ as:

$$
\alpha_{f} \widehat{\mathbf{U}}_{i-1}+\widehat{\mathbf{U}}_{i}+\alpha_{f} \widehat{\mathbf{U}}_{i+1}=\sum_{l=0}^{3} \frac{\gamma_{l}}{2}\left(\mathbf{U}_{i+l}+\mathbf{U}_{i-l}\right)
$$

where $\alpha_{f}=0.47$, and $\gamma_{l}$ are the filter coefficients [13]. The filter is employed on a uniformly spaced grid thanks to a coordinate transform. For LES computations, the filter also plays the role of a subgrid-scale model, relaxing turbulent energy at high frequencies [22-24]. Time integration is performed by applying a low-storage 6-stage Runge Kutta algorithm [2]. Radiation boundary conditions, Navier-Stokes characteristic boundary conditions, and sponge zones are used in order to avoid significant acoustic reflections at the mesh boundaries. A more detailed description of the numerical algorithm is given in Fosso et al. [14].

\subsection{Reconstruction for conforming grid interfaces}

2.3.1. Numerical scheme At the mesh-block interfaces, the implicit centered scheme (11) used in the computation of the convective fluxes cannot be applied. Thus, in a previous study [12], a flux reconstruction technique has been proposed at the interfaces of conforming grids. It is presented in the following by considering a two-dimensional computational domain composed of two blocks $\mathrm{L}$ and R separated by a conforming interface, as shown in Fig. 3.

\section{(a)}

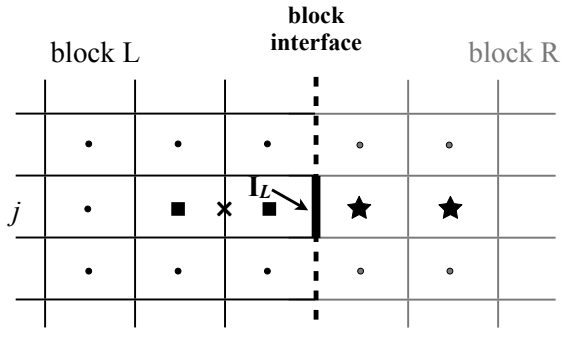

$i=N-2 \quad i=N-1 \quad i=N \quad i^{\prime}=0 \quad i^{\prime}=1$ (b)

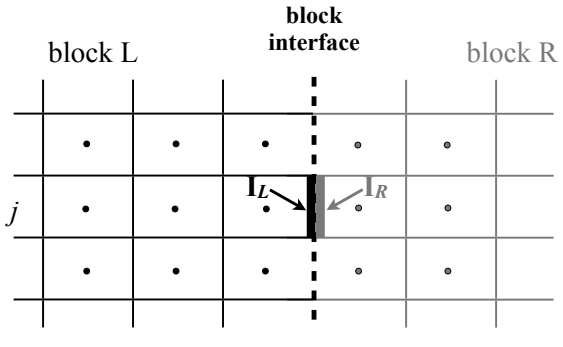

$i=N-2 \quad i=N-1 \quad i=N \quad i^{\prime}=0 \quad i^{\prime}=1$

Figure 3. Flux reconstruction for conforming grids: (a) step 1: computation of the flow variables at the interface $\boldsymbol{I}_{\boldsymbol{L}}$ using a scheme involving two cells (squares) and an interface (cross) of block $\mathrm{L}$ and two ghost cells (stars) of block R, (b) step 2: flux computation from the flow variables at the interfaces $\boldsymbol{I}_{\boldsymbol{L}}$ and $\boldsymbol{I}_{\boldsymbol{R}}$, using a Riemann solver.

The reconstruction technique consists of two steps. In the first step, the flow variables $\widetilde{\mathbf{U}}$ at the grid interface in blocks $\mathrm{L}$ and $\mathrm{R}$ are determined using upwind schemes. More precisely, in block L, as illustrated in Fig. 3(a), the vector $\widetilde{\mathbf{U}}_{L}$ at the interface $I_{L}$ is computed using a non-centered scheme involving the flow variables in cells of blocks $\mathrm{L}$ and $\mathrm{R}$ such as:

$$
\alpha^{\prime} \widetilde{\mathbf{U}}_{i=N-1 / 2, j}+\widetilde{\mathbf{U}}_{L}=\underbrace{a_{0}^{\prime} \mathbf{U}_{i=N-1, j}+a_{1}^{\prime} \mathbf{U}_{i=N, j}}_{\text {cells of block L }}+\underbrace{a_{2}^{\prime} \mathbf{U}_{i^{\prime}=0, j}+a_{3}^{\prime} \mathbf{U}_{i^{\prime}=1, j}}_{\text {cells of block R }}
$$

where $\alpha^{\prime}$ and $a_{i}^{\prime}$ are the scheme coefficients determined using Taylor series. For block $\mathrm{L}$, the values of $\mathbf{U}$ in the cells $\left(i^{\prime}=0, j\right)$ and $\left(i^{\prime}=1, j\right)$ of block $\mathbf{R}$ are a priori not known. These cells are thus referred to as ghost cells for block $\mathrm{L}$ in the following. The values of $\mathbf{U}$ in the ghost cells are obtained thanks to data exchanges between the blocks at each time iteration of the simulation. Symmetrically, 


\section{to}

The flux reconstruction for conforming grids presented in this section has been successfully applied

$$
\widetilde{\mathbf{U}}_{R}+\beta^{\prime \prime} \widetilde{\mathbf{U}}_{i^{\prime}=1 / 2, j}=\underbrace{a_{0}^{\prime \prime} \mathbf{U}_{i=N-1, j}+a_{1}^{\prime \prime} \mathbf{U}_{i=N, j}}_{\text {cells of block L }}+\underbrace{a_{2}^{\prime \prime} \mathbf{U}_{i^{\prime}=0, j}+a_{3}^{\prime \prime} \mathbf{U}_{i^{\prime}=1, j}}_{\text {cells of block R }}
$$

where $\beta^{\prime \prime}$ and $a_{i}^{\prime \prime}$ are the scheme coefficients. The values of $\widetilde{\mathbf{U}}_{L}$ and $\widetilde{\mathbf{U}}_{R}$ are usually not identical, since they are determined from two different upwind schemes (13) and (14). Therefore, in a second step, a Riemann problem [25] is solved in order to ensure the unicity of the flux, hence the scheme conservativity, at the block interface.

2.3.2. Selective filter In the vicinity of conforming grid interfaces, as for the centered scheme (11), the 7-point centered filter (12) cannot be applied, and its formulation has to be modified. However, previous studies [26] demonstrated that the change of the filter formulation at the grid interface is likely to significantly decrease the accuracy of the filtering process and generate spurious noise. Therefore, in order to still apply the centered filter (12) at the grid interface, Fosso [26] proposed to artificially extend the size of the mesh blocks using ghost cells, and to modify the filter formulation in the ghost cell regions exclusively. In practice, according to the notations of Fig. 4, in order to change the filter formulation as far as possible from the interface, the block $\mathrm{L}$ is extended using five ghost cells represented by stars. These cells correspond to the cells of block $\mathrm{R}$ indexed by $i^{\prime}=0,1,2,3,4$. Consequently, in block $\mathrm{L}$, the centered filter (12) on 7 points can be applied in cells $i=\ldots, N-1, N$ and in the ghost cell $\left(i^{\prime}=0, j\right)$. Finally, a non-centered filter is used in order to determine the value of $\widehat{\mathbf{U}}$ in the ghost cell $\left(i^{\prime}=1, j\right)$ in grey in Fig. 4:

$\alpha_{f} \widehat{\mathbf{U}}_{i^{\prime}=0, j}+\widehat{\mathbf{U}}_{i^{\prime}=1, j}=\sum_{k=2}^{3} \frac{\gamma_{k}}{2} \mathbf{U}_{i=N-k+2, j}+\frac{\gamma_{1}}{2} \mathbf{U}_{i^{\prime}=0, j}+\gamma_{0} \mathbf{U}_{i^{\prime}=1, j}+\sum_{k=1}^{3} \frac{\gamma_{k}}{2} \mathbf{U}_{i^{\prime}=k+1, j}-\alpha_{f} \mathbf{U}_{i^{\prime}=2, j}$

to massively parallel aeroacoustic simulations of jet flows at high Reynolds numbers [14, 15, 17].

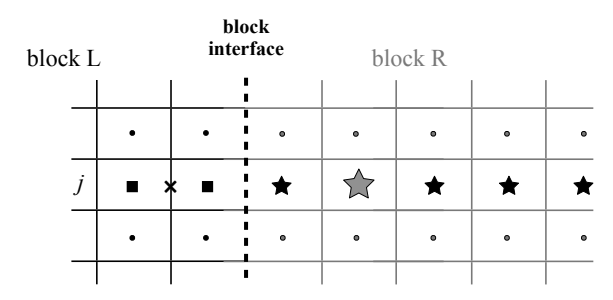

$i=N-1 \quad i=N \quad i^{\prime}=0 \quad i^{\prime}=1 \quad i^{\prime}=2 \quad i^{\prime}=3 \quad i^{\prime}=4$

Figure 4. Filter application in block $\mathrm{L}$ in the vicinity of a conforming grid interface: the flow variables in the ghost cell $i^{\prime}=1$ in grey are filtered using grid cells of block L (squares) and block R (stars).

\section{FLUX RECONSTRUCTION TECHNIQUE FOR NON-CONFORMING GRIDS}

In this section, the reconstruction presented above for conforming grids is extended to the cases of plane and curved non-conforming meshes. 


\subsection{Plane interfaces}

In the case of a non-conforming grid interface, as illustrated in Fig. 5, the flux reconstruction technique described in section 2.3 cannot be used. Indeed, for such grids, as the mesh lines are discontinuous across the block interface, the ghost cells represented by stars in Fig. 4 are not defined anymore. Therefore, the upwind schemes (13) (14) and the filter (15) cannot be applied. In this work, a new flux reconstruction is thus proposed at the non-conforming interfaces. It consists in using noncentered schemes and meshless interpolations in order to define the flow variables in ghost cells and at the grid interface. In this section, the flux reconstruction is presented for the plane grid interface displayed in Fig. 5, considering block $\mathrm{L}$ as the current block.

3.1.1. Numerical scheme In order to compute the flux at the interface $I_{L}$ in block L, the key idea is to make possible the application of the schemes (13) and (14) thanks to the reconstruction of the flow variables in ghost cells. For this purpose, a methodology, composed of four steps depicted in Fig. 5, is presented. In step 1, two ghost cells, represented in grey in Fig. 5(a), are defined. The centers of these cells, depicted by stars, are located at the intersection between the mesh lines $i^{\prime}=0$ and $i^{\prime}=1$ and the straight line passing by the centers of the cells $(i=N-1, j)$ and $(i=N, j)$. The values of the flow variables $\mathbf{U}$ in the ghost cells are determined from the values of $\mathbf{U}$ in the cells of block $\mathrm{R}$ using a meshless interpolation. The interpolation technique is presented in section 3.1.3. In step 2, illustrated in Fig. 5(b), the upwind scheme (13) can be applied to compute the flow vector $\widetilde{\mathbf{U}}_{L}$ at the interface $I_{L}$ of block L. In step 3, symmetrically with what was done in steps 1 and 2 for block L, ghost cells are defined in block R and the scheme (14) is employed to determine the vector $\widetilde{\mathbf{U}}$ at the interfaces $\left(\ldots, I_{R, j^{\prime}}, I_{R, j^{\prime}+1}, \ldots\right)$ in grey in Fig. 5(c). Finally, in step 4, a ghost interface $I_{L}^{\prime}$, identical geometrically to $I_{L}$, is defined in block R, as shown in Fig. 5(d). The variable vector $\widetilde{\mathbf{U}}_{L}^{\prime}$ at the interface $I_{L}^{\prime}$ is interpolated from the values $\widetilde{\mathbf{U}}$ obtained in step 3 . This second interpolation method is also described in section 3.1.3. Even if the interfaces $I_{L}^{\prime}$ and $I_{L}$ are geometrically identical, the values of $\widetilde{\mathbf{U}}$ at these two interfaces differ since they are computed from different schemes and interpolations. Therefore, the convective flux at the block interface is determined from the values of $\widetilde{\mathbf{U}}_{L}$ and $\widetilde{\mathbf{U}}_{L}^{\prime}$ by resolving a Riemann flux problem [25].

3.1.2. Selective filter Five ghost cells are necessary in order to apply the non-centered filter (15) near the block interface. For conforming grids, as explained in section 2.3, the flow variables in the ghost cells are directly obtained thanks to data exchanges between blocks. However, for non-conforming grids, these variables first need to be interpolated before being exchanged. Consequently, using five ghost cells results in an extra computational cost compared to conforming grids, which led us to only consider two ghost cells. The application of the filter close to the nonconforming interface of block L is illustrated in Fig. 6. The cells and ghost cells of block L involved in the filtering are represented by squares and stars, respectively. As shown in Fig. 6(a), using two ghost cells, the 7-point centered filter (12) can be applied as far as point $(i=N-1, j)$ in block L. At the cell $(i=N, j)$ adjacent to the block interface, in grey in Fig. 6(b), the filtered field $\widehat{\mathbf{U}}_{i=N, j}$ is computed from the upwind formulation:

$$
\alpha_{f} \widehat{\mathbf{U}}_{i=N-1, j}+\widehat{\mathbf{U}}_{i=N, j}+\alpha_{f} \widehat{\mathbf{U}}_{i^{\prime}=0, j}=\sum_{k=0}^{4} \gamma_{k}^{\prime} \mathbf{U}_{N-4+k, j}+\gamma_{5}^{\prime} \mathbf{U}_{i^{\prime}=0, j}+\gamma_{6}^{\prime} \mathbf{U}_{i^{\prime}=1, j}
$$


(a) step 1

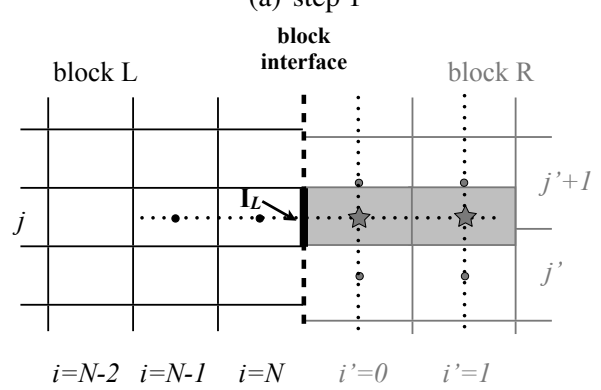

(c) $\operatorname{step} 3$

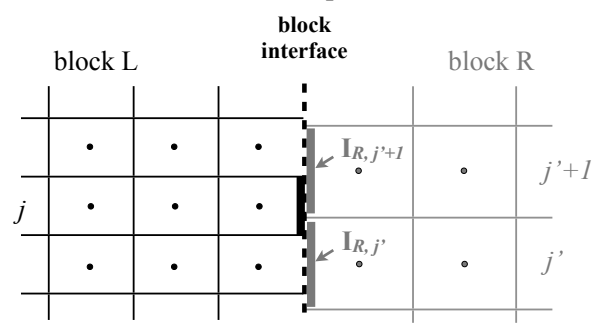

$i=N-2 \quad i=N-1 \quad i=N \quad i^{\prime}=0 \quad i^{\prime}=1$ (b) step 2

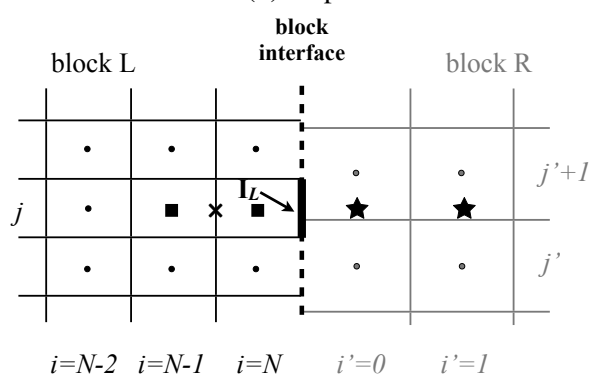

(d) $\operatorname{step} 4$

block

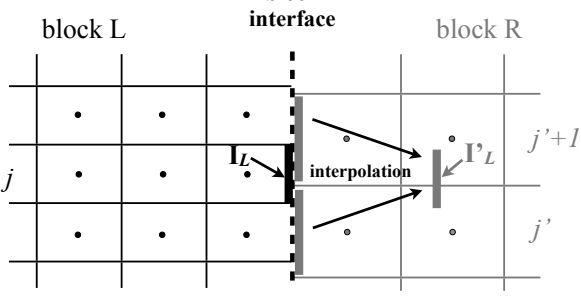

$i=N-2 \quad i=N-1 \quad i=N \quad i^{\prime}=0 \quad i^{\prime}=1$

Figure 5. Flux reconstruction for non-conforming grids at the interface $I_{L}$ in block L: (a) step 1: definition of two ghost cells (stars), (b) step 2: computation of the flow variables at the interface $\boldsymbol{I}_{\boldsymbol{L}}$ using a non-centered scheme involving an interface (cross) and two cells (squares) of block L, and two ghost cells (stars), (c) step 3: computation of the flow variables at the interfaces in grey, applying steps 1 and 2 in block R, (d) step 4: interpolation of the flow variables at the ghost interface $\boldsymbol{I}_{\boldsymbol{L}}^{\prime}$ using the data computed in step 3 , and computation of the resulting flux at the block interface using a Riemann solver.

\subsubsection{Interpolation techniques In the flux reconstruction for non-conforming grids, interpolations} are performed in order to compute the flow variables $\mathbf{U}$ in two ghost cells and the values of $\widetilde{\mathbf{U}}$ at the grid interface. As presented in section 3.1.1, in block $\mathrm{L}$, the interpolations are carried out using values of $\mathbf{U}$ and $\widetilde{\mathbf{U}}$ in block R. In practice, block $\mathrm{R}$ can be divided into subdomains with a loss of topology information between the domains. Therefore, in this study, meshless interpolations based on Radial Basis Functions (RBF) are employed.

First, the interpolation technique is described for the calculation of a component $u$ of the vector $\mathbf{U}$ in the ghost cell located at $i^{\prime}=0$ in Fig. 5(a). The calculation is performed using the value of $u$ known in $n_{v}$ cells of block $\mathrm{R}$ surrounding the ghost cell. These $n_{v}$ cells are located along the line $i^{\prime}=0$ for a 2-D mesh, in the plane $i^{\prime}=0$ for a 3-D straight mesh. The RBF approximation $u_{\mathrm{RBF}}$ of 
(a)

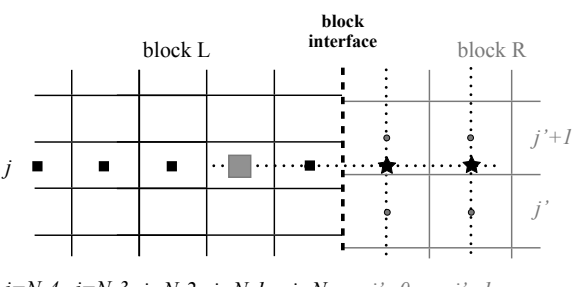

$i=N-4 \quad i=N-3 \quad i=N-2 \quad i=N-1 \quad i=N \quad i^{\prime}=0 \quad i^{\prime}=1$

(c)

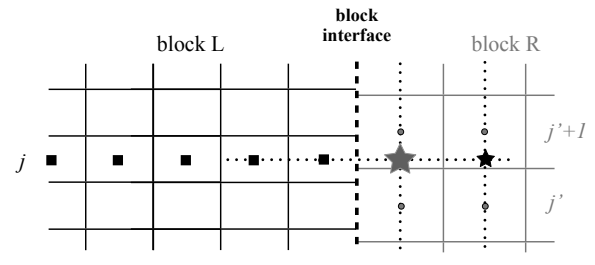

$i=N-4 \quad i=N-3 \quad i=N-2 \quad i=N-1 \quad i=N \quad i^{\prime}=0 \quad i^{\prime}=1$ (b)

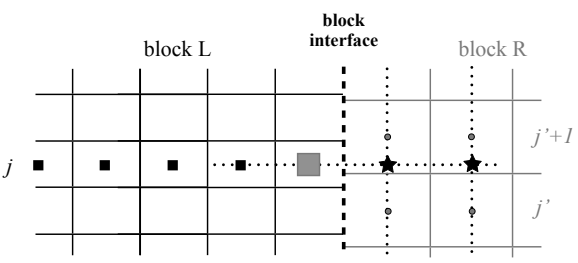

$i=N-4 \quad i=N-3 \quad i=N-2 \quad i=N-1 \quad i=N \quad i^{\prime}=0 \quad i^{\prime}=1$

(d)

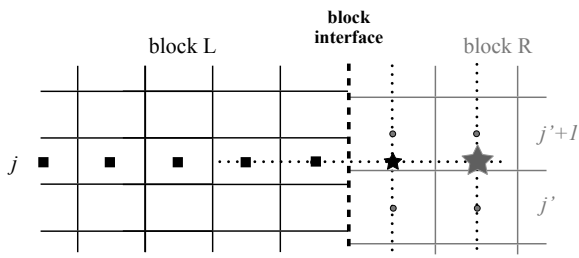

$i=N-4 \quad i=N-3 \quad i=N-2 \quad i=N-1 \quad i=N \quad i^{\prime}=0 \quad i^{\prime}=1$

Figure 6. Filtering at the non-conforming grid interface in block L. Cells in block L (squares) and ghost cells in block $\mathrm{R}$ (stars) used in the filter scheme applied at points: (a) $(i=N-\mathbf{1}, j),(\mathrm{b})(\boldsymbol{i}=\boldsymbol{N}, \boldsymbol{j}),(\mathrm{c})$ $\left(i^{\prime}=0, j\right)$ and $(\mathrm{d})\left(i^{\prime}=1, j\right)$.

the variable $u$ at point $\boldsymbol{x}$ writes [9]:

$$
u_{\mathrm{RBF}}(\boldsymbol{x})=\sum_{j=1}^{n_{v}} \xi_{j} \Phi\left(\boldsymbol{x}, \boldsymbol{x}_{j}\right)+\sum_{q=1}^{m} \zeta_{q} P_{q}(\boldsymbol{x})
$$

where $\xi_{j}$ and $\zeta_{q}$ are the unknown interpolation coefficients, $\left(\boldsymbol{x}_{j}\right)_{j=1, \ldots, n_{v}}$ are the centers of the $n_{v}$ cells, $\Phi$ are Wendland's radial basis functions [9, 28], and $\sum \zeta_{q} P_{q}(\boldsymbol{x})=\zeta_{0}+\zeta_{1} x+\zeta_{2} y+\ldots+\zeta_{m} z^{\operatorname{deg}(\mathrm{P})}$ is a polynomial term of degree $\operatorname{deg}(\mathrm{P})$ that ensures the unicity of the approximation $u_{\mathrm{RBF}}[18,29]$. The calculation of the coefficients $\xi_{j}$ and $\zeta_{q}$ is presented in appendix A.1. Similarly, the value of $\mathbf{U}$ in the second ghost cell in Fig. 5(a) is interpolated using the RBF approximation (18) and $n_{v}$ points located at $i^{\prime}=1$. The choice of the interpolation parameters $n_{v}$ and $\operatorname{deg}(\mathrm{P})$ in Eq. (18) is discussed in sections 6 and 7.

A second interpolation technique is proposed in order to interpolate the flow variables $\widetilde{\mathbf{U}}$ at the block interface $I_{L}^{\prime}$ in Fig. 5(d). As for the interpolation of the flow field in the ghost cells, a RBF interpolation is carried out. However, the quantity to interpolate is not a single-point value $u$ but an averaged value $\widetilde{u}$ on a grid interface. Therefore, the interpolation of $\widetilde{u}$ on the interface $I_{L}^{\prime}$ is performed from $n_{v}$ values of $\widetilde{\mathbf{U}}$ at the interfaces $\left(I_{R, 1}, \ldots, I_{R, j^{\prime}}, I_{R, j^{\prime}+1}, \ldots, I_{R, n_{v}}\right)$ represented in grey in Fig. 5(c). The interpolation formulation at the grid interface $I_{L}^{\prime}$ is obtained by integrating Eq (18) on $I_{L}^{\prime}$ :

$$
\begin{aligned}
\tilde{u}_{L^{\prime}} & =\frac{1}{\left|I_{L}^{\prime}\right|} \int_{I_{L}^{\prime}} u_{\mathrm{RBF}}(\boldsymbol{x}) \mathrm{d} \boldsymbol{x} \\
& =\sum_{j^{\prime}=1}^{n_{v}} \widetilde{\xi}_{j^{\prime}}\left(\frac{1}{\left|I_{L}^{\prime}\right|} \int_{I_{L}^{\prime}} \Phi\left(\boldsymbol{x}, \boldsymbol{x}_{j^{\prime}}\right) \mathrm{d} \boldsymbol{x}\right)+\sum_{q=1}^{m} \widetilde{\zeta}_{q}\left(\frac{1}{\left|I_{L}^{\prime}\right|} \int_{I_{L}^{\prime}} P_{q}(\boldsymbol{x}) \mathrm{d} \boldsymbol{x}\right)
\end{aligned}
$$


where the point $\boldsymbol{x}_{j^{\prime}}$ is the center of the surface $I_{R, j^{\prime}}$. The calculation of the interpolation coefficients $\widetilde{\xi}_{j^{\prime}}$ and $\widetilde{\zeta}_{q}$ is detailed in appendix A.2.1. A 3rd-order Gaussian quadrature is used in order to compute the integrals of Eq. (19). In practice, the interpolation coefficients in Eq. (18) and (19) are computed only once at the beginning of the simulation and stored in memory, yielding low CPU cost interpolations (see appendix A).

\subsection{Curved interfaces}

For curved grid interfaces, the flux reconstruction presented in section 3.1 cannot be applied. For the interpolation of the flow variables at the grid interface using Eq. (19), as the curvature of the surface is not taken into account to define the ghost interface $I_{L}^{\prime}$, the integral (19) is evaluated on a plane interface that does match the shape of the non-conforming interface. Therefore, a flux reconstruction for curved non-conforming interfaces is also proposed. The objective is to find a function $\sigma(x)$ to define a curved interface $I_{L_{\text {curved }}}^{\prime}$ knowing only the position $x$ of the mesh points. The flow variables at the interface $I_{L_{\text {curved }}}^{\prime}$ are then calculated as in Eq. (19):

$$
\begin{aligned}
\tilde{u}_{L^{\prime}} & =\frac{1}{\left|I_{L_{\text {curved }}}^{\prime}\right|} \int_{I_{L_{\text {curved }}^{\prime}}} u_{\mathrm{RBF}}(\boldsymbol{x}) \mathrm{d} \boldsymbol{x} \\
& =\sum_{j^{\prime}=1}^{n_{v}} \widetilde{\xi}_{j^{\prime}}\left(\frac{1}{\left|I_{L_{\text {curved }}}^{\prime}\right|} \int_{I_{L_{\text {curved }}^{\prime}}} \Phi\left(\boldsymbol{x}, \boldsymbol{x}_{j^{\prime}}\right) \mathrm{d} \boldsymbol{x}\right)+\sum_{q=1}^{m} \widetilde{\zeta}_{q}\left(\frac{1}{\left|I_{L_{\text {curved }}}^{\prime}\right|} \int_{I_{L_{\text {curved }}^{\prime}}} P_{q}(\boldsymbol{x}) \mathrm{d} \boldsymbol{x}\right)
\end{aligned}
$$

The calculation of the RBF coefficients $\widetilde{\xi}_{j^{\prime}}$ and $\widetilde{\zeta}_{q}$ is described in appendix A.2.2.

The method to determine the function $\sigma$ is presented for the 2-D grid of Fig. 7(a), composed of two blocks $\mathrm{L}$ and $\mathrm{R}$ separated by a curved interface. The curved interfaces to be defined by the function $\sigma$ are denoted by $I_{L_{\text {curved }}}^{\prime}$ and $\left(I_{R, 1_{\text {curved }}}, \ldots, I_{R, j_{\text {curved }}^{\prime}}, I_{R, j^{\prime}+1_{\text {curved }}}, \ldots, I_{R, n_{v_{\text {curved }}}}\right)$. In order to determine the function $\sigma$, a technique proposed by Carr et al. [30] for 3-D imaging reconstruction is employed. First, relations to be verified by the function $\sigma$ at given mesh points are imposed. In particular, at the $N_{p}$ grid points of blocks $\mathrm{L}$ and $\mathrm{R}$ lying on the interfaces $\left(I_{R, 1_{\text {curved }}}, \ldots, I_{R, j_{\text {curved }}^{\prime}}, I_{R, j^{\prime}+1_{\text {curved }}}, \ldots, I_{R, n_{v_{\text {curved }}}}\right)$ in Fig. 7(a), the function $\sigma$ cancels out:

$$
\sigma\left(\boldsymbol{x}_{i}\right)=0 \quad 1 \leq i \leq N_{p}
$$

where $\boldsymbol{x}_{i}=\left(x_{i}, y_{i}, z_{i}\right)$ are the spatial coordinates of the $i$ th-mesh point. In order to ensure that function $\sigma$ differs from the zero-function, off-surface points are considered and non-zero values are given to the function $\sigma$ at these points. In the present study, $n_{v}$ points of block $\mathrm{L}$ and $n_{v}$ points of block R are selected, corresponding to the centers of the cells adjacent to the grid interface. They are represented by black and grey circles in Fig. 7(b) for $n_{v}=3$. A value of $\sigma=-1$ is given to the $n_{v}$ points of block $\mathrm{L}$, and $\sigma=1$ is attributed to the $n_{v}$ points of block R. Thus, the objective is to find the function $\sigma$ so that the following relations are satisfied:

$$
\begin{cases}\sigma\left(\boldsymbol{x}_{i}\right)=0 & 1 \leq i \leq N_{p} \\ \sigma\left(\boldsymbol{x}_{r}\right)=1 & 1 \leq r \leq n_{v} \\ \sigma\left(\boldsymbol{x}_{l}\right)=-1 & 1 \leq l \leq n_{v}\end{cases}
$$


where $\left(\boldsymbol{x}_{r}\right)_{1, \ldots, n_{v}}$ and $\left(\boldsymbol{x}_{l}\right)_{1, \ldots, n_{v}}$ are the positions of the centers of the $n_{v}$ cells of blocks $\mathrm{L}$ and $\mathrm{R}$, respectively. Then, given the set of points $S_{N}=\left[\left(\boldsymbol{x}_{i}\right)_{1, . ., N_{p}},\left(\boldsymbol{x}_{r}\right)_{1, . ., n_{v}},\left(\boldsymbol{x}_{l}\right)_{1, . ., n_{v}}\right]=\left[\left(\boldsymbol{x}_{j}\right)_{1, . ., N_{S}}\right]$ and the relations (22), the function $\sigma$ is calculated by RBF interpolation [30]:

$$
\sigma(\boldsymbol{x}) \simeq \sigma_{\mathrm{RBF}}(\boldsymbol{x})=\sum_{j=1}^{N_{S}} \Theta_{j} \Phi\left(\boldsymbol{x}, \boldsymbol{x}_{j}\right)+\sum_{q=1}^{m} \kappa_{q} P_{q}(\boldsymbol{x})
$$

where $N_{S}=N_{p}+2 n_{v}$, and $\Theta_{j}$ and $\kappa_{q}$ are the unknown interpolation coefficients computed similarly as for the ghost cells (see appendix A.1).

(a)

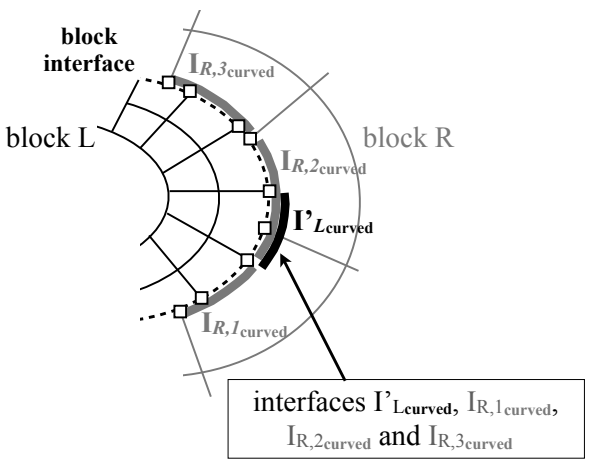

(b)

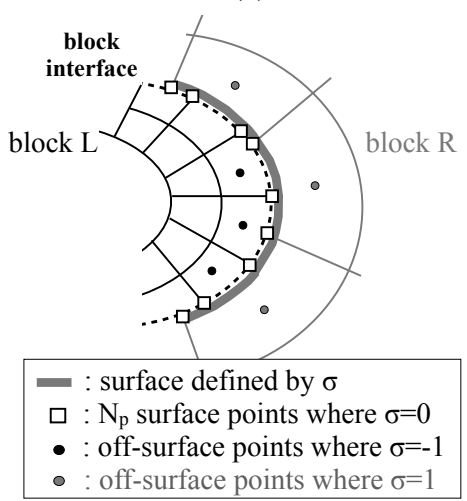

Figure 7. Computation of the function $\sigma$ that defines the curved interfaces $I_{L_{\text {curved }}}^{\prime}$ and $\left(I_{R, j_{\text {curved }}^{\prime}}\right)_{1 \leq j^{\prime} \leq n_{v}=3}$ : (a) interfaces $I_{L_{\text {curved }}}^{\prime}$ and $\left(I_{R, j_{\text {curved }}^{\prime}}\right)_{1 \leq j^{\prime} \leq 3}$ and $N_{p}$ points (squares) lying on the interfaces, (b) surface points (squares) where $\sigma=0$ and off-surface points (circles) where $\sigma \neq 0$.

\section{PROPERTIES OF THE RBF INTERPOLATION IN THE WAVENUMBER SPACE}

The performance of the RBF interpolation is evaluated in the wavenumber space. For this purpose, a uniform 1-D mesh extending over the range [0,1], composed of 81 points $\left(x_{j}\right)_{1 \leq j \leq 81}$ is considered:

$$
x_{j}=(j-1) \Delta \text { for } 1 \leq j \leq 81
$$

with $\Delta=1 / 80$. At the points $x_{j}$, a harmonic function $f_{k}(x)=\exp (\mathrm{i} k x)$ is imposed, where $k$ is the wavenumber with $k \Delta$ varying from 0 to $\pi$, and $\mathrm{i}$ is the complex number verifying $\mathrm{i}^{2}=-1$. For RBF interpolations, a second 1-D mesh, referred to as the RBF grid, is defined using $N_{\mathrm{RBF}}=41$ points located at the following positions:

$$
x_{j}^{\prime}=\left(0.2 \Delta+\frac{j-1}{N_{\mathrm{RBF}}-1}\right) \quad \text { for } \quad 1 \leq j \leq N_{\mathrm{RBF}}
$$

In this way, the distance between two consecutive RBF grid points is equal to $2 \Delta$ and there is a full point-mismatch between the two 1-D meshes. The interpolation of $f_{k}$ on the RBF grid is denoted $g_{k}$ in the following. For consistency with the finite-volume flux reconstruction proposed in this study, the function $g_{k}$ is defined over each segment $\left[x_{j}^{\prime}, x_{j+1}^{\prime}\right]$, with $j \in\left[1, N_{\mathrm{RBF}}-1\right]$. For $x \in\left[x_{j}^{\prime}, x_{j+1}^{\prime}\right]$, 
from Eq. (18), the function $g_{k}$ writes as follows:

$$
g_{k}(x)=\sum_{l=1}^{n_{v}} \xi_{l} \Phi\left(x, x_{l}\right)+P(x)
$$

The $n_{v}$ nearest mesh points $\left(x_{l}\right)$ that surround point $x_{j_{\mathrm{M}}}^{\prime}=\left(x_{j}^{\prime}+x_{j+1}^{\prime}\right) / 2$ and where the values of $f_{k}$ are known are used to determine the interpolation coefficients in Eq. (26). In this section, the influence of the number of points $n_{v}$ is evaluated by performing interpolations using $n_{v}=4$, 6,8 and 20 points. The interpolations are carried out using the 2 nd-degree polynomial function $P(x)=\zeta_{0}+\zeta_{1} x+\zeta_{2} x^{2}$, where $\left(\zeta_{j}\right)_{0 \leq j \leq 2}$ are the unknown interpolation coefficients. The influence of the degree of $P$ on the accuracy of the spatial discretization is discussed in section 5 .

First, the accuracy of the RBF interpolation is examined. For this purpose, an interpolation error $\epsilon$ is computed as a function of the wavenumber $k$ from the difference between the values of $f_{k}$ and $g_{k}$ over each segment $\left[x_{j}^{\prime}, x_{j+1}^{\prime}\right]$ as:

$$
\epsilon(k)=\sum_{j=1}^{N_{\mathrm{RBF}}-1} \int_{x_{j}^{\prime}}^{x_{j+1}^{\prime}}\left|f_{k}(x)-g_{k}(x)\right| \mathrm{d} x
$$

where $|\cdot|$ is the complex modulus. Secondly, the energy of the interpolated signal $g_{k}$ is compared with the energy of the original signal $f_{k}$ through the evaluation of the integrals $E_{f}$ and $E_{g}$ defined as:

$$
E_{f}(k)=\int_{x_{1}^{\prime}}^{x_{N_{\mathrm{RBF}}}^{\prime}}\left|f_{k}(x)\right|^{2} \mathrm{~d} x=1 \quad \text { and } \quad E_{g}(k)=\int_{x_{1}^{\prime}}^{x_{N_{\mathrm{RBF}}^{\prime}}}\left|g_{k}(x)\right|^{2} \mathrm{~d} x
$$

For comparison, interpolations are also performed using the polynomial functions of degrees 2 and 3 given by:

$$
\begin{aligned}
& P_{2}(x)=c_{1}+c_{2} x+c_{3} x^{2} \\
& P_{3}(x)=c_{4}+c_{5} x+c_{6} x^{2}+c_{7} x^{3}
\end{aligned}
$$

where $\left(c_{j}\right)_{1 \leq j \leq 7}$ are the interpolation coefficients. Note that, as for the RBF interpolations, the polynomial approximations (29) and (30) are defined by pieces over each segment $\left[x_{j}^{\prime}, x_{j+1}^{\prime}\right]$. The interpolations coefficients $\left(c_{i}\right)$ are determined using a least-square approximation involving $n_{v}$ nearest points surrounding point $x_{j_{\mathrm{M}}}^{\prime}$. More precisely, over each segment $\left[x_{j}^{\prime}, x_{j+1}^{\prime}\right]$, the values of $\left(c_{i}\right)$ are calculated to minimize the functions $\chi_{P_{2}}$ and $\chi_{P_{3}}$ :

$$
\begin{aligned}
\chi_{P_{2}}\left(c_{1}, c_{2}, c_{3}\right) & =\sum_{l=1}^{n_{v}=4} \frac{\left|P_{2}\left(x_{l}\right)-f_{k}\left(x_{l}\right)\right|^{2}}{\left(x_{l}-x_{j_{\mathrm{M}}}^{\prime}\right)^{2}} \\
\chi_{P_{3}}\left(c_{4}, c_{5}, c_{6}, c_{7}\right) & =\sum_{l=1}^{n_{v}=6} \frac{\left|P_{3}\left(x_{l}\right)-f_{k}\left(x_{l}\right)\right|^{2}}{\left(x_{l}-x_{j_{\mathrm{M}}}^{\prime}\right)^{2}}
\end{aligned}
$$

The variations of the energy $E_{g}$ obtained from the RBF interpolations using $n_{v}=4,6,8$ and 20 points and from the polynomial interpolations with $P_{2}$ and $P_{3}$ are represented in Fig. 8(a) as a function of the normalized wavenumber $k \Delta$. When RBF is used, the value of $E_{g}$ decreases with $k \Delta$, 
indicating higher levels of dissipation at high wavenumbers. The highest levels of dissipation are obtained using $n_{v}=4$. In particular, for $k \Delta=\pi / 3$, the energy is equal to 0.995 for $n_{v}=4$ whereas values $0.99<E_{g}<1$ are obtained for $n_{v}=6,8$ and 20. The dissipation obtained using RBF is lower than that calculated from a polynomial interpolation of degree 2 over all the wavenumber range. In addition, using RBF, no energy amplification is observed whereas energy values $E_{g}>1$ are found using polynomial interpolation $P_{3}$ for $\pi / 8 \leq k \Delta \leq \pi / 2$ in Fig. 8(a). Therefore, it is interesting to use RBF to preserve the energy stability and to maintain low dissipation levels for wavenumbers $k \Delta<\pi / 4$, which are well resolved by the present spatial discretization schemes.

The interpolation errors $\epsilon$ obtained using RBF and polynomial interpolations are represented in Fig. 8(b) as a function of the wavenumber $k \Delta$. When RBF is used, the highest values of $\epsilon$ are obtained for $n_{v}=4$. In this case, the interpolation error is stronger than that calculated with the polynomial interpolation $P_{3}$ for $k \Delta<\pi / 2$. However, it is lower than the error computed with $P_{2}$ which involves the same number of interpolation points. When the number of interpolation points $n_{v}$ increases, as expected, the value of $\epsilon$ decreases all over the wavenumber range. For $n_{v}=20$, as a result, the error $\epsilon$ is lower than the error computed using $P_{3}$ for $\pi / 12<k \Delta<\pi$. For $k \Delta<\pi / 12$, it is higher than that obtained using $P_{3}$, but is very small and lower than $5 \times 10^{-6}$.

(a)

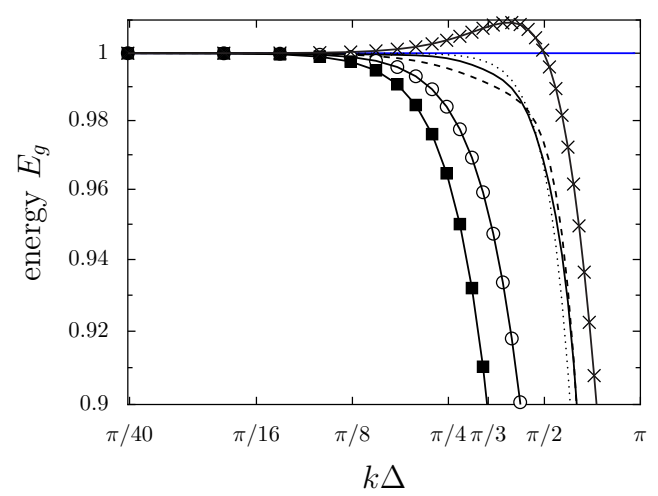

(b)

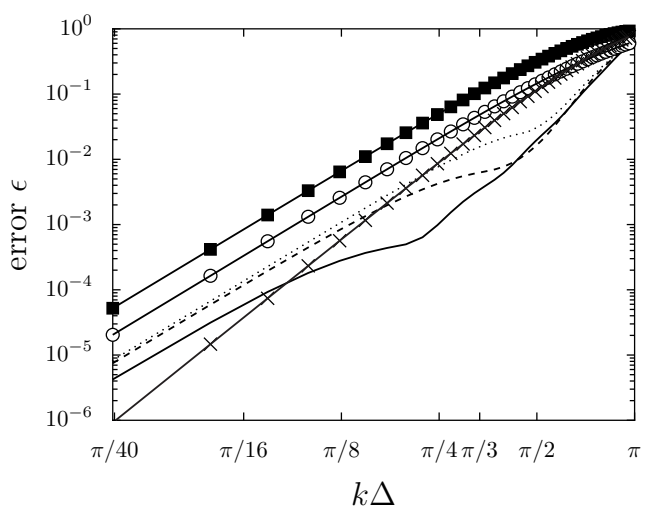

Figure 8. Representation of (a) the energy $E_{g}$ and (b) the interpolation error $\epsilon$ as a function of the normalized wavenumber $k \Delta$ : RBF interpolations using $\circ n_{v}=4, \cdots n_{v}=6,---n_{v}=8,-n_{v}=20$, polynomial interpolations of $\boldsymbol{a}$ degree 2 and $\times$ degree $3 .-E_{g}=E_{f}=1$.

\section{ACOUSTIC PULSE}

In order to examine the overall accuracy of the flux reconstruction presented for non-conforming grids above, an acoustic pulse is imposed in the vicinity of a non-conforming interface in a medium at rest. For this purpose, the two-dimensional domain of size $\ell \times \ell$ shown in Fig. 9(a) is considered, with $\ell=100 \mathrm{~m}$. It is composed of two blocks separated by a non-conforming interface located at 
$x=0.6 \ell$. At $t=0$, the pulse is introduced at $x_{p}=0.4 \ell$ and $y_{p}=0.5 \ell$ as:

$$
\left\{\begin{array}{l}
\rho^{\prime}(x, y)=A_{p} \exp \left(-\ln 2 \frac{\left(x-x_{p}\right)^{2}+\left(y-y_{p}\right)^{2}}{\hbar^{2}}\right) \\
u^{\prime}(x, y)=v^{\prime}(x, y)=0 \\
p^{\prime}(x, y)=c_{0}^{2} \rho^{\prime}(x, y)
\end{array}\right.
$$

where $\hbar=0.03 \ell$ is the pulse half-width, $A_{p}$ is the pulse amplitude, and $c_{0}$ is the ambient sound speed. The ambient pressure and temperature are equal to $p_{0}=10^{5} \mathrm{~Pa}$ and $T_{0}=300 \mathrm{~K}$, respectively. Radiation boundary conditions and sponge layers are used. An exact solution of the problem can be derived from the linearized Euler equations [31]. In order to compare the numerical results obtained from the Navier-Stokes equations with the exact solution, an amplitude $A_{p}$ of $0.1 \mathrm{~Pa}$ is chosen. In addition, the viscous terms in Eq. (1) are neglected in the simulations.

The performance of the flux reconstruction is evaluated using six Cartesian grids referred to as pulsegrid1, pulsegrid2, pulsegrid3, pulsegrid4, pulsegrid5 and pulsegrid6, and two flux reconstruction techniques with and without RBF interpolation. The RBF interpolations are carried out using a number of $n_{v}=8$ points. The influence of the degree $\operatorname{deg}(\mathrm{P})$ of the RBF polynomial function in Eq. (18) is examined using polynomial functions of degree 0,1 and 2. For the flux reconstruction without RBF, 2nd-order interpolations are used in order to reconstruct the flow variables in the ghost cells and at the grid interface [32]. This reconstruction, available in the els $\boldsymbol{A}$ solver [20], is described in appendix A.3.

The mesh parameters, namely the grid spacings $\Delta x$, and the grid spacings $\Delta y^{L}$ and $\Delta y^{R}$ at the left and right hand sides of the block interface are given in Tab. I. The meshes pulsegrid2, pulsegrid3, pulsegrid4, pulsegrid5 and pulsegrid6 are respectively $2,3,4,5$ and 6 times finer than pulsegrid1. In all cases, at the left-hand side of the interface, a uniform grid spacing $\Delta x=\Delta y^{L}$ is used in the directions $x$ and $y$. For pulsegrid1, it is equal to $0.02 \ell$. At the right hand-side of the interface, the grid spacing is also equal to $\Delta x$ in $x$ direction whereas the mesh spacing $\Delta y^{R}$ is twice larger than $\Delta y^{L}$ in $y$ direction. In order to have a full point-mismatch at the grid interface, for $x>0.6 \ell$, in all cases, the grid cells are shifted upwards of $0.5 \Delta y^{L}$.

\begin{tabular}{cccc}
\hline mesh & $\Delta x$ & $\Delta y^{L}$ & $\Delta y^{R}$ \\
\hline pulsegrid1 & $\ell / 50$ & $\ell / 50$ & $\ell / 25$ \\
pulsegrid2 & $\ell / 100$ & $\ell / 100$ & $\ell / 50$ \\
pulsegrid3 & $\ell / 150$ & $\ell / 150$ & $\ell / 75$ \\
pulsegrid4 & $\ell / 200$ & $\ell / 200$ & $\ell / 100$ \\
pulsegrid5 & $\ell / 250$ & $\ell / 250$ & $\ell / 125$ \\
pulsegrid6 & $\ell / 300$ & $\ell / 300$ & $\ell / 150$ \\
\hline
\end{tabular}

Table I. Mesh spacings used in the simulations of the pulse.

The time step $\Delta t$ of the simulations is chosen sufficiently small so that the errors related to the time discretization are negligible. More precisely, its value is calculated in order to provide a CFL number $c_{0} \Delta t / \Delta x$ of 0.05 for $\Delta x=\ell / 300$. The fluctuating pressure field $p^{\prime}$ obtained at $t=1200 \Delta t$ using pulsegrid 2 is represented in Fig. 9(a). At this instant, the acoustic wave reaches the non-conforming interface. In order to evaluate the effective order of the spatial discretization in the presence of the non-conforming interface, the pressure fluctuation obtained at $t=1200 \Delta t$ is 
compared to the exact solution $p_{\text {exact }}^{\prime}$ through the $L_{2}$ relative error:

$$
\epsilon_{p}=\left(\frac{\int_{\Omega_{\epsilon}}\left(p^{\prime}-p_{\text {exact }}^{\prime}\right)^{2} \mathrm{~d} \Omega}{\int_{\Omega_{\epsilon}} p_{\text {exact }}^{\prime 2} \mathrm{~d} \Omega}\right)^{1 / 2}
$$

where $\Omega_{\epsilon}=\left\{(x, y) \in \mathbb{R}^{2} \mid 0.2 \ell \leq x, y \leq 0.8 \ell\right\}$. In finite-volume, $p^{\prime}$ is the averaged value of the fluctuating pressure over each cell of domain $\Omega_{\epsilon}$ (see Eq. (8)). Therefore, for consistency, the exact solution is calculated similarly. The discrete form of Eq. (34) thus writes as:

$$
\epsilon_{p}=\left(\frac{\sum_{\text {cell } \in \Omega_{\epsilon}}\left(p^{\prime}-p_{\text {exact cell }}^{\prime}\right)^{2}}{\sum_{\text {cell } \in \Omega_{\epsilon}} p_{\text {exact cell }}^{2}}\right)^{1 / 2}
$$

where $p_{\text {exact cell }}^{\prime}=\left(1 /\left|\Omega_{\text {cell }}\right|\right) \int_{\Omega_{\text {cell }}} p_{\text {exact }}^{\prime} \mathrm{d} \Omega$ and $\left|\Omega_{\text {cell }}\right|$ is the volume of the cell. Simulations with four uniform Cartesian meshes without grid interfaces with grid spacings of $\Delta x=\Delta y=\ell / 50$, $\ell / 100, \ell / 200$ and $\ell / 300$ respectively have also been done for comparisons.

The errors $\epsilon_{p}$ obtained using the non-conforming grids with and without the flux reconstruction based on RBF for polynomial functions of degrees 0, 1 and 2 are presented in Fig 9(b), as a function of the grid spacing $\Delta x / \ell$. Those obtained using the meshes without grid interface are also indicated. In all cases, the amplitude of $\epsilon_{p}$ decreases as the value of $\Delta x$ tends to 0 . Using the grid without interface, the error profile follows a 6th-order convergence slope. This result is expected since the present spatial discretization is based on 6th-order numerical schemes (see Fosso et al. [12]). With non-conforming interfaces, the 6th-order convergence slope is not retrieved, and higher error levels are obtained for $\Delta x \leq 0.005 \ell$ compared to the simulations without interfaces. The stronger errors are obtained using the flux reconstruction without RBF, with an error profile varying following a 2nd-order slope. When RBF is used, lower errors are obtained, and they decrease with the degree of $P$. In particular, the error profile calculated with $\operatorname{deg}(\mathrm{P})=2$ is in $\operatorname{good}$ agreement with that obtained for conforming grids. In the following, the RBF interpolations are therefore performed using $\operatorname{deg}(\mathrm{P})=2$.

(a)

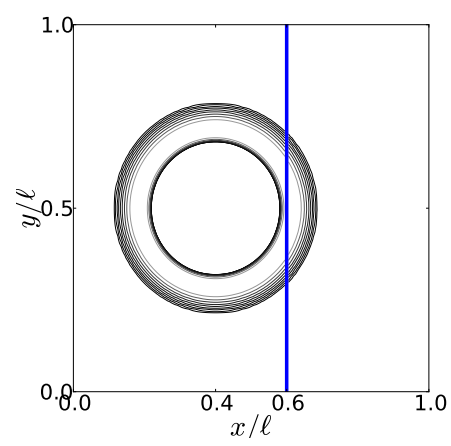

(b)

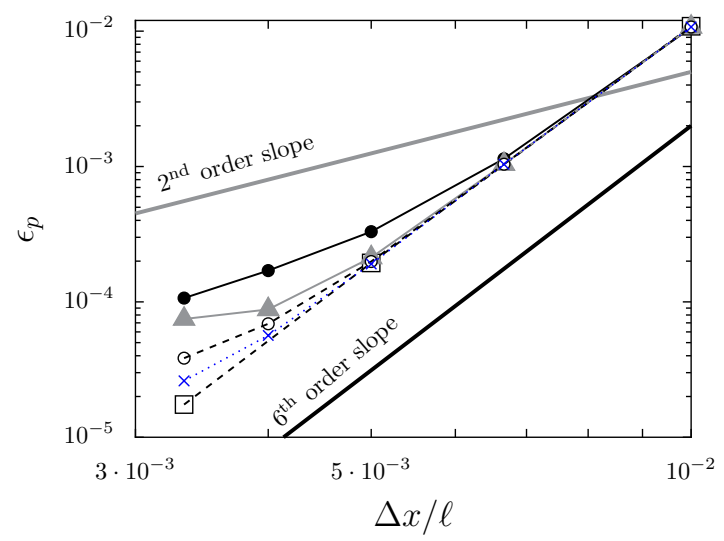

Figure 9. (a) Fluctuating pressure $p^{\prime}$ at $t=1200 \Delta t$ using pulsegrid2, with 10 isocontours from $10^{-4}$ to $10^{-2} \mathrm{~Pa}$ following a geometric progression of ratio 1.67. The non-conforming interface is shown in blue. (b) Error profiles $\epsilon_{p}$ as a function of the grid spacing $\Delta x / \ell$ : RBF interpolations with polynomial functions $\Delta \operatorname{deg}(\mathrm{P})=0, \circ \operatorname{deg}(\mathrm{P})=1, \times \operatorname{deg}(\mathrm{P})=2, \bullet$ interpolation without $\mathrm{RBF}$ and $\square$ grids without interface. 


\section{CONVECTION OF A VORTEX} given by: $t=0$.

$$
\left\{\begin{aligned}
u^{\prime} & =-\frac{\Gamma}{R^{2}}\left(y-y_{c}\right) \exp \left(-\ln 2 \frac{\left(x-x_{c}\right)^{2}+\left(y-y_{c}\right)^{2}}{2 b^{2}}\right) \\
v^{\prime} & =\frac{\Gamma}{R^{2}}\left(x-x_{c}\right) \exp \left(-\ln 2 \frac{\left(x-x_{c}\right)^{2}+\left(y-y_{c}\right)^{2}}{2 b^{2}}\right) \\
p^{\prime} & =-\frac{\rho \Gamma^{2}}{2 R^{2}} \exp \left(-\ln 2 \frac{\left(x-x_{c}\right)^{2}+\left(y-y_{c}\right)^{2}}{b^{2}}\right)
\end{aligned}\right.
$$

where $\left(x_{c}=0.5 L, y_{c}=0.5 L\right)$ is the position of the vortex center at the initial time $t=0$, $b=(\sqrt{\ln 2} / 20) L \simeq 0.04 L$ is the vortex Gaussian half-width, and $\Gamma$ represents the vortex intensity

$$
\frac{\rho \Gamma^{2}}{2 R^{2}}=10^{3} \mathrm{~Pa}
$$

where $R=b / \sqrt{\ln 2}$. The velocity and pressure fluctuations are superimposed onto the mean flow at

The performance of the flux reconstruction is examined by performing simulations using four meshes referred to as Finegrid, Mediumgrid, Coarsegrid, and Verycoarsegrid, and two flux reconstruction techniques with and without RBF interpolations. When RBF is applied, the influence of the number of interpolation points $n_{v}$ is studied by carrying out interpolations with $n_{v}=4$, 6,8 and 12 points. The RBF interpolations are performed using a second-degree polynomial function in Eq. (18). The influence of the degree of the polynomial function has been examined by performing simulations using polynomial functions of degrees 0,1 and 2 . The use of the seconddegree polynomial function provided the lowest spurious noise levels at the grid interface. For the sake of concision, these results are not presented in this study. Views of the four meshes close to the block interface are given in Fig. 10. The mesh parameters, including the grid spacings $\Delta x$ in the streamwise direction, and the grid spacings $\Delta y^{L}$ and $\Delta y^{R}$ at the left and the right sides of the block interface are provided in Tab. II. In all cases, in the streamwise direction, a grid spacing of $\Delta x=\Delta=L / 255$ is used. The vortex half-width $b$ is thus discretized by 10 points, given that $b=10.6 \Delta$. In the transverse direction, on the left hand side of the interface, the grid spacing $\Delta y^{L}$ is equal to $\Delta$. On the right hand side, the mesh resolution in the $y$ direction is different from $\Delta$. More precisely, the grid spacing $\Delta y^{R}$ is respectively equal to $0.5 \Delta, 2 \Delta, 4 \Delta$ and $6 \Delta$ for Finegrid, Mediumgrid, Coarsegrid and Verycoarsegrid, corresponding to a discretization of the vortex halfwidth by $21.2,5.3,2.6$ and 1.8 points. In addition, in order to ensure a full point-mismatch at the 
(a)

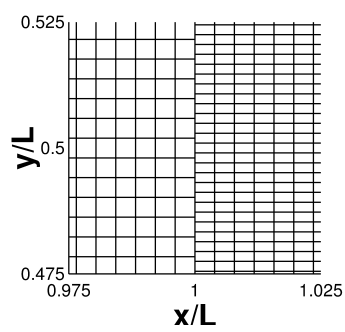

(b)

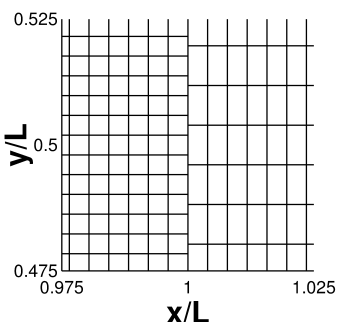

(c)

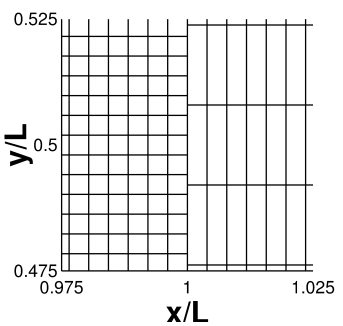

(d)

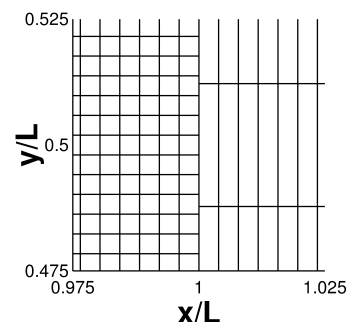

Figure 10. Representation of the meshes close to the block interface: (a) Finegrid, (b) Mediumgrid, (c) Coarsegrid, and (d) Verycoarsegrid.

\begin{tabular}{cccc}
\hline mesh & $\Delta x$ & $\Delta y^{L}$ & $\Delta y^{R}$ \\
\hline Finegrid & $\Delta$ & $\Delta$ & $\mathbf{0 . 5} \Delta$ \\
Mediumgrid & $\Delta$ & $\Delta$ & $\mathbf{2} \Delta$ \\
Coarsegrid & $\Delta$ & $\Delta$ & $\mathbf{4} \Delta$ \\
Verycoarsegrid & $\Delta$ & $\Delta$ & $\mathbf{6} \Delta$
\end{tabular}

Table II. Mesh spacings for the Fine, Medium, Coarse, and Verycoarse grids.

The time step $\Delta t$ in the computations is chosen in order to impose a CFL number $(1+M) c_{0} \Delta t / \Delta$ of 0.4 , where $c_{0}$ is the ambient sound speed. When the vortex crosses the block interface, spurious waves are generated due to the difference in grid resolution as well as to the specific spatial discretization at the interface. The objective here is to ensure that the amplitude of these spurious waves is very low with respect to the pressure deficit in the vortex. For that, the pressure field $p_{\text {interface }}$ obtained in the multiblock simulations is compared with the pressure field $p_{\text {no-interface }}$ computed from a simulation without block interface. That monoblock simulation is carried out using the same computational domain with mesh spacings $\Delta x=\Delta y=\Delta$. By comparing the pressure $p_{\text {interface }}$ with $p_{\text {no-interface }}$ instead of with the analytical vortex solution (36), the error thus obtained only results from the effects of the non-conforming grid and not from discretization errors. In addition, the pressure field differences $\Delta p=p_{\text {interface }}-p_{\text {no-interface }}$ are only computed at the left side of the block interface where the mesh is similar in the two computations. In this way, the pressure fields $p_{\text {interface }}$ and $p_{\text {no-interface }}$ are computed at the same point. In particular, the time evolution of $\Delta p$ is recorded at the two mesh points $\mathrm{A}$ and $\mathrm{B}$, indicated by squares in Fig. 11. They are located, respectively, at the interface at $x=L$ and $y=0.5 L$, and upstream of the block interface at $x=0.8 \mathrm{~L}$ and $y=0.75 \mathrm{~L}$. The signal recorded at point A provides information on the vortex deformation at the block interface, while the signal at point $\mathrm{B}$ gives the amplitude of the spurious waves propagating from the interface.

6.1.1. Grid sensitivity The influence of the mesh resolution is evaluated by performing four simulations using Finegrid, Mediumgrid, Coarsegrid and Verycoarsegrid. The simulation settings are given in Tab. III. In the four simulations, the flux reconstruction at the block interface is 


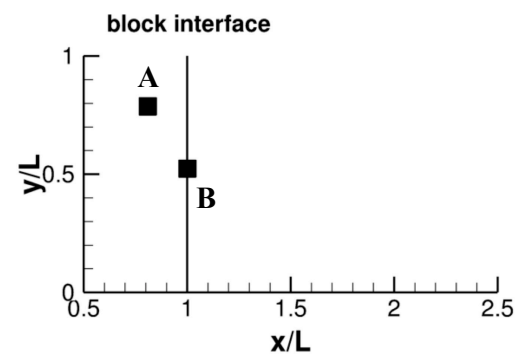

Figure 11. Representation of the mesh points A and B (squares) where the pressure field is recorded.

performed using RBF, with interpolations on $n_{v}=8$ points and the second-degree polynomial function $\left(P_{q}\right)_{(1, . ., m)}=\left(1, x, y, x^{2}, y^{2}, x y\right)$.

\begin{tabular}{cccc}
\hline mesh & flux reconstruction technique & $n_{v}$ & RBF polynomial degree \\
\hline Finegrid & RBF & 8 & 2 \\
Mediumgrid & RBF & 8 & 2 \\
Coarsegrid & RBF & 8 & 2 \\
Verycoarsegrid & RBF & 8 & 2 \\
\hline
\end{tabular}

Table III. Parameters of the simulations in the grid sensitivity study.

The time evolution of the pressure $|\Delta p|=\left|p_{\text {interface }}-p_{\text {no-interface }}\right|$ recorded at points $\mathrm{A}$ and $\mathrm{B}$ in the simulations is presented in Fig. 12, where $|\cdot|$ is the absolute value. The vertical blue line in the figures indicates the moment when the vortex hits the block interface. The signal amplitudes are displayed in log scale in order to enhance the differences between the simulations. At point A, in Fig. 12(a), the maximum value of $|\Delta p|$ is obtained at the instant when the vortex crosses the interface in all cases. Using Verycoarsegrid, the pressure fluctuation peak is equal to $28.1 \mathrm{~Pa}$, corresponding to $2.7 \%$ of the pressure at the center of the vortex. Using Coarsegrid, the pressure difference reaches a value of $5 \mathrm{~Pa}$. Using the medium and the refined meshes, the amplitudes of the spurious waves at point $\mathrm{A}$ are significantly lower than those found for the coarse grids, and do not exceed $0.9 \mathrm{~Pa}$ and $0.2 \mathrm{~Pa}$, respectively. At point B in Fig 12(b), the noise level also decreases as the mesh is refined at the right hand side of the block interface. Indeed, maximum pressure differences of $3.6 \mathrm{~Pa}, 0.4 \mathrm{~Pa}$, $0.1 \mathrm{~Pa}$ and $0.03 \mathrm{~Pa}$ are obtained in Verycoarsegrid, Coarsegrid, Mediumgrid and Finegrid. These levels are much lower than those at point A. Note that using Verycoarsegrid, the vortex half-width $b$ is only discretized by 1.8 points at the right side of the block interface. As a consequence, the mesh is not fine enough and the vortex structure is strongly modified when it crosses the block interface, yielding $|\Delta p|>0.5 \mathrm{~Pa}$ at points $\mathrm{A}$ and $\mathrm{B}$ for $t>10000 \Delta t$. These results demonstrate that nonconforming grids must be designed such that the flow field is correctly discretized at both sides of the interface. In the present simulations, given the vortex Gaussian half-width $b$, a grid spacing $\Delta y^{R} \leq 4 \Delta$ is recommended, corresponding to a discretization of the half-width $b$ by 2.6 points (i.e 5.2 points in the vortex width). This result was expected since the numerical methods used in this study well-calculate the scales discretized by at least 5 points per wavelength [14]. Let us mention that values $\Delta y^{R}>4 \Delta$ could be used in sponge zones, that is to say in flow regions close to the domain boundaries where the mesh is deliberately coarse in order to damp hydrodynamic fluctuations before they reach the boundary conditions. 
(a)

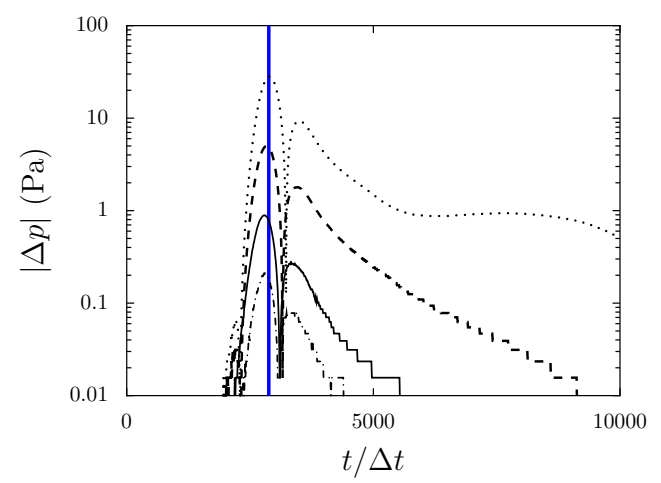

(b)

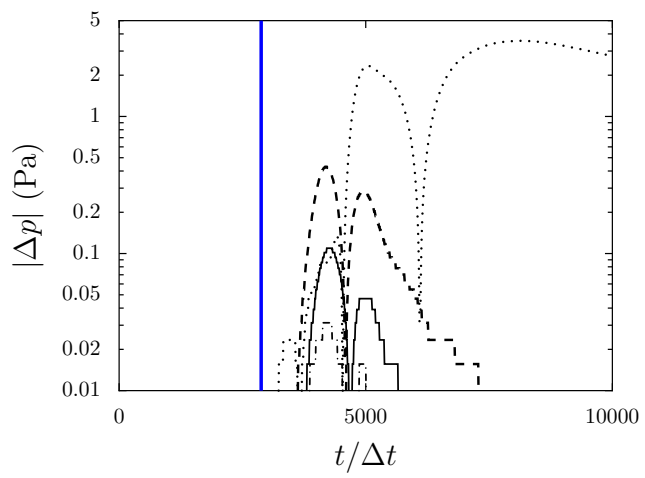

Figure 12. Representation of the time evolution of the pressure difference $|\Delta p|=\left|p_{\text {interface }}-p_{\text {no-interface }}\right|$ (a) at point A and (b) at point B: - · - Finegrid, _ Mediumgrid, - - - Coarsegrid, and .... Verycoarsegrid. The vertical blue line indicates the moment when the vortex hits the interface. 
(a)

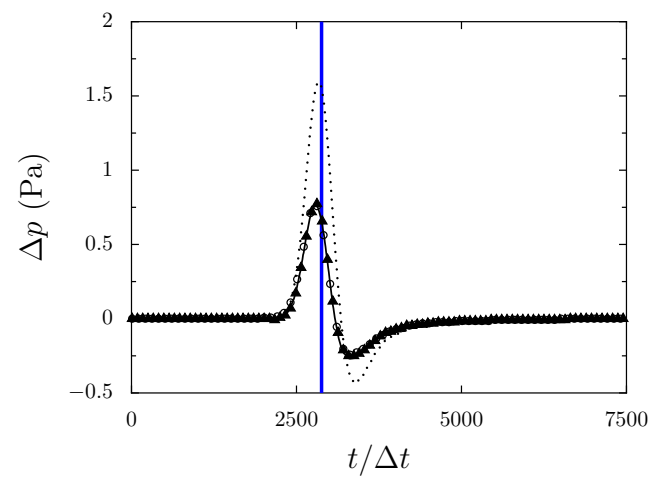

(b)

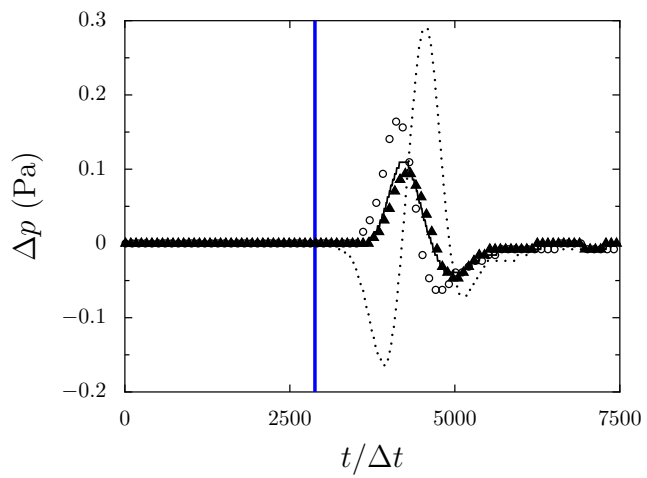

Figure 13. Representation of the time evolution of the pressure difference $\Delta p=p_{\text {interface }}-p_{\text {no-interface }}$ (a) at point $\mathrm{A}$ and (b) at point $\mathrm{B}: \cdots n_{v}=4, \circ n_{v}=6, \boldsymbol{\Delta} n_{v}=8$ and $-n_{v}=12$ points. The vertical blue line indicates the moment when the vortex hits the interface.

(a)

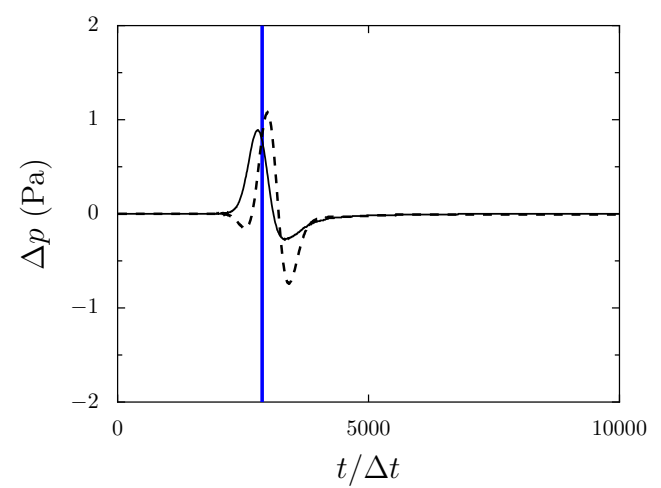

(b)

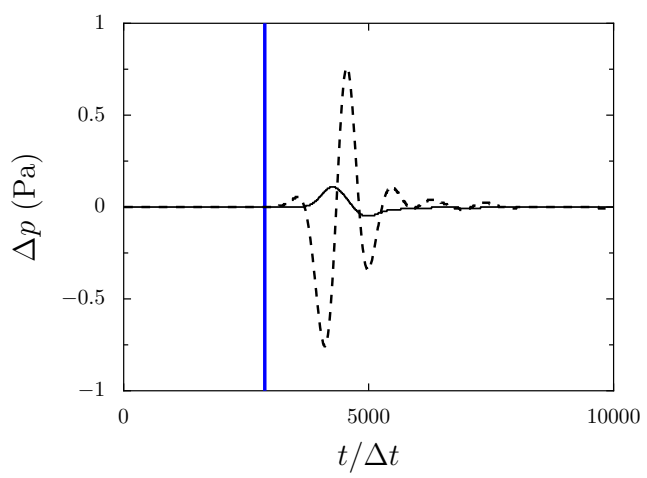

Figure 14. Representation of the time evolution of the pressure difference $\Delta p=p_{\text {interface }}-p_{\text {no-interface }}$ (a) at point $A$ and (b) at point B using Mediumgrid: — RBF, - - - no RBF. The vertical blue line indicates the moment when the vortex hits the interface. 
(a)

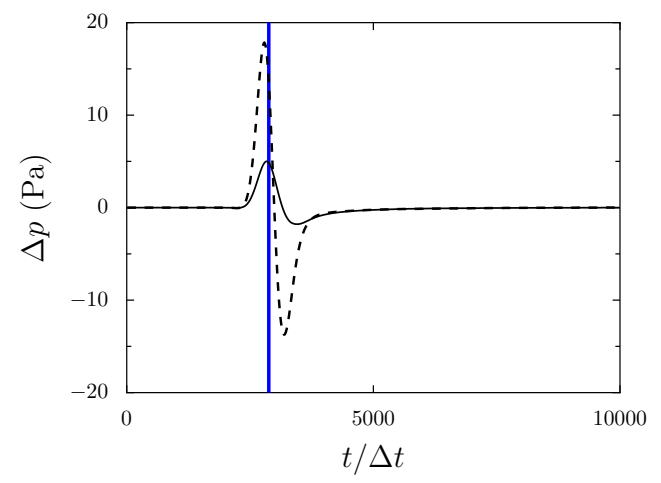

(b)

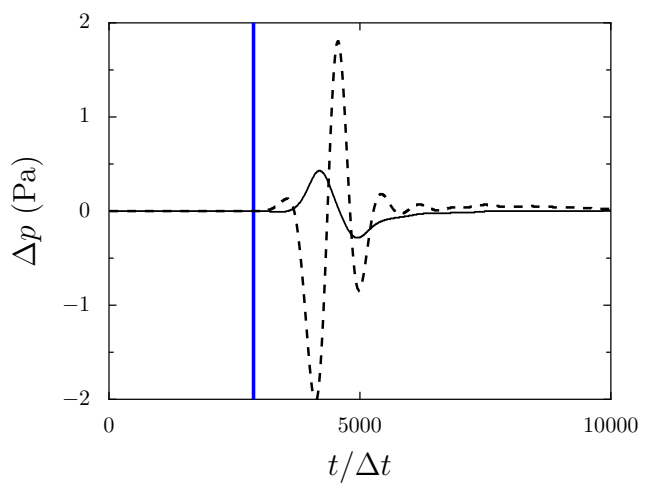

Figure 15. Representation of the time evolution of the pressure difference $\Delta p=p_{\text {interface }}-p_{\text {no-interface }}$ (a) at point $\mathrm{A}$ and (b) at point $\mathrm{B}$ using Coarsegrid: — RBF, - - - no RBF. The vertical blue line indicates the moment when the vortex hits the interface.

\subsection{Wavy grids}

In order to examine the performance of the flux reconstruction for curved non-conforming interfaces, the vortex defined in section 6.1 is convected on 2-D wavy grids. Three computational domains, presented in Fig. 16, are considered. They are composed of 2 blocks separated by a wavy non-conforming interface located close to $x=L$, where $L=0.1 \mathrm{~m}$. The wavy grid interfaces are defined by a sinusoïdal shape of wavelength $\lambda_{x}$ and of amplitude $\lambda_{y}$. The values of $\lambda_{x}$ and $\lambda_{y}$ are provided in Tab. VI for the different meshes. In the grid referred to as wavy1, the block interface has a height of $\lambda_{y}=24 b$ and a sinusoïdal shape of amplitude of $\lambda_{x}=8 b$, where $b$ is the vortex halfwidth. In wavy2, the amplitude of the sinusoïdal interface is two times higher than in wavy1, i.e. $\lambda_{x}=16 b$, but $\lambda_{y}=24 b$ as previously. In wavy3, the block interface is composed of three sinusoïdal arches with $\lambda_{x}=\lambda_{y}=8 b$. In all cases, a grid spacing $\Delta x=\Delta=L / 127$ is used in the $x$ direction, leading to a vortex half-width discretized by 5.3 points. In the $y$ direction, the grid spacing is equal to $\Delta y^{L}=\Delta$ at the left hand side of the interface. In order to create non-conforming grids, a mesh spacing $\Delta y^{R}=L / 87$ is applied at the right hand side of the interface, yielding $b=2.6 \Delta$. The vortex, convected from the left to the right, is initially located at $y_{0}=0.5 \mathrm{~L}$, and at equal distance from the domain inlet and the block interface in the $x$ direction.

\begin{tabular}{ccc}
\hline mesh & $\lambda_{x}$ & $\lambda_{y}$ \\
\hline wavy1 & $8 b$ & $24 b$ \\
wavy2 & $16 b$ & $24 b$ \\
wavy3 & $8 b$ & $8 b$ \\
\hline
\end{tabular}

Table VI. Parameters of the wavy grid interfaces.

Six simulations are performed using wavy1, wavy 2 and wavy3, and the flux reconstructions designed for plane and curved interfaces. Their parameters are given in Tab. VII. In all cases, RBF interpolations are carried out using a number of $n_{v}=8$ points and the second-degree polynomial function. The time step $\Delta t$ in the computations is chosen such that CFL number $(1+M) c_{0} \Delta t / \Delta=0.2$. 
(a)

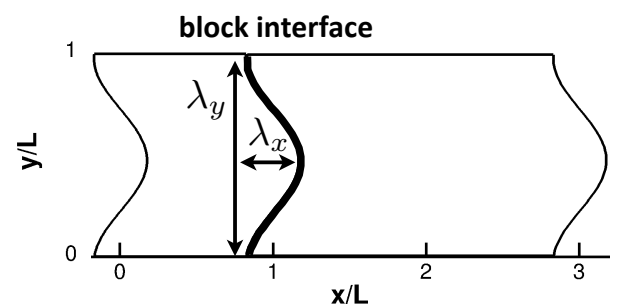

(b)

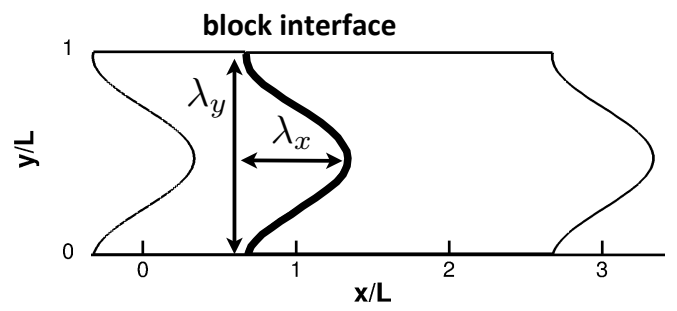

(c)

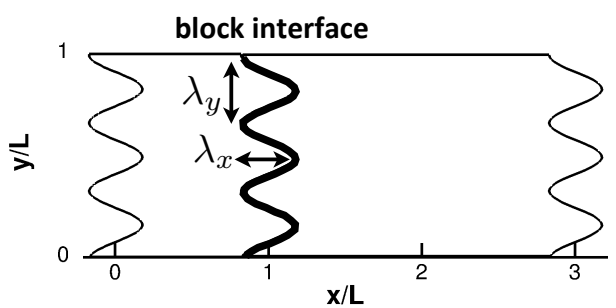

Figure 16. Representation of the wavy computational domains: (a) wavy1, (b) wavy2, and (c) wavy3. The non-conforming interface is indicated by a bold line. 
(a)

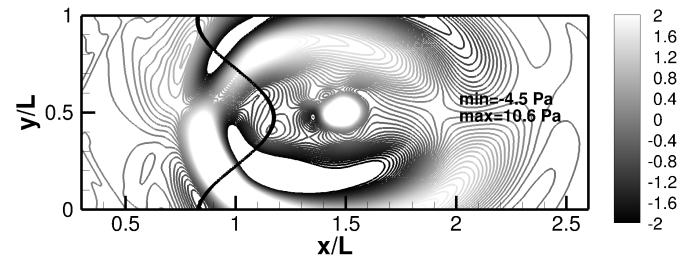

(b)

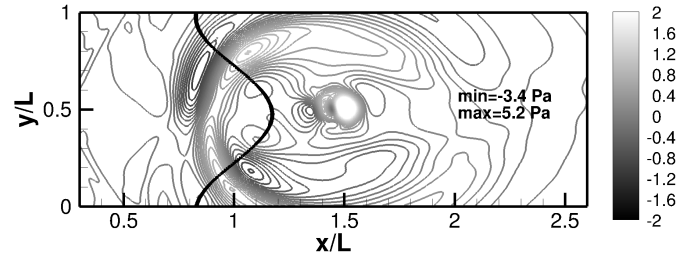

Figure 17. Representation of the pressure difference $\Delta p$ at $t=2800 \Delta t$ using wavy1: flux reconstruction for (a) plane interfaces and (b) curved interfaces, levels given in $\mathrm{Pa}$.

The pressure difference $\Delta p$ obtained at $t=2800 \Delta t$ using wavy2 is plotted in Fig. 18. Noise levels of 10-20 Pa are found. They are higher compared to the results obtained using wavy1 in Fig. 17. This is due to the block interface which displays stronger variations than that using wavyl. The pressure difference obtained in Fig. 18 (a) with the flux reconstruction technique for curved interfaces shows weaker pressure wave amplitudes compared to the pressure difference obtained in Fig. 18 (b) for the plane interface reconstruction.

(a)

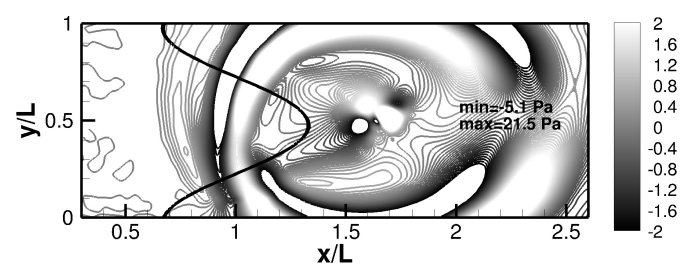

(b)

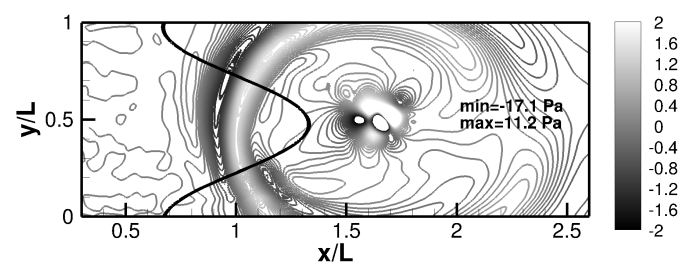

Figure 18. Representation of the pressure difference $\Delta p$ at $t=2800 \Delta t$ using wavy2: flux reconstruction for (a) plane interfaces and (b) curved interfaces, levels given in $\mathrm{Pa}$.

Snapshots of the pressure difference $\Delta p$ obtained using wavy3 at $t=2800 \Delta t$ are displayed in Fig. 19. As for wavy1 and wavy2, lower spurious noise is found using the curved reconstruction technique than the plane one. However, the use of a block interface with 3 arches generates higher spurious noise levels than previously, with maximum values of $\Delta p$ of $200 \mathrm{~Pa}$ reached at this instant. In particular, the vortex core, located at $x=1.5 \mathrm{~L}$ is strongly affected by the presence of the block interface. The use of non-conforming interfaces with low curvature therefore seems to be recommended.

(a)

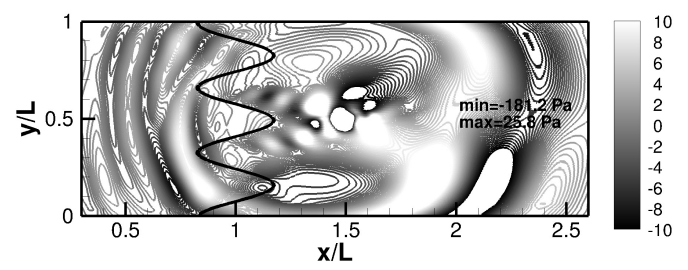

(b)

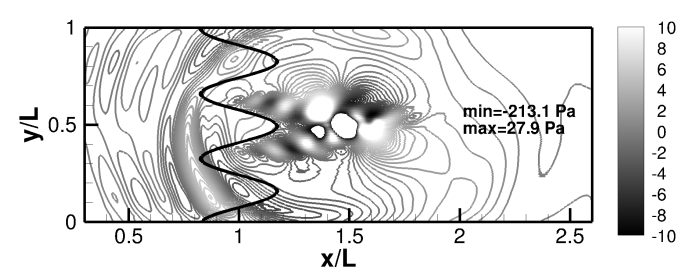

Figure 19. Representation of the pressure difference $\Delta p$ at $t=2800 \Delta t$ using wavy3: flux reconstruction for (a) plane interfaces and (b) curved interfaces, levels given in $\mathrm{Pa}$. 


\section{THREE-DIMENSIONAL JET}

In order to demonstrate the applicability of the flux reconstruction technique to a 3-D simulation, a circular isothermal jet is computed using a Cartesian mesh with a non-conforming interface. The aim is to prove that the use of non-conforming interfaces does not significantly affect the jet flow development and the sound field radiated by the jet in the near-field region.

\subsection{Jet definition}

The jet flow has a Mach number of $M=u_{j} / c_{0}=0.9$ and a Reynolds number of $\operatorname{Re}_{D}=u_{j} D / \nu=4 \times 10^{5}$, corresponding to the conditions of the jet in the numerical simulation of Bogey and Bailly [33], where $D$ and $u_{j}$ are the jet diameter and velocity, $c_{0}$ is the sound speed and $\nu$ is the molecular viscosity. The ambient pressure $p_{0}$ and temperature $T_{0}$ are respectively equal to $10^{5} \mathrm{~Pa}$ and $300 \mathrm{~K}$. The jet inflow, located at $x=0$, is characterized by the mean longitudinal velocity profile given by the hyperbolic tangent-profile:

$$
u(r)=\frac{1}{2} u_{j}\left(1+\tanh \left(\frac{r_{0}-r}{2 \delta_{\theta}}\right)\right)
$$

where $\delta_{\theta}=r_{0} / 20$ is the initial momentum thickness of the shear layer, $r_{0}=D / 2$ is the jet radius, and $r=\sqrt{y^{2}+z^{2}}$. The mean density profile is computed from a Crocco-Busemann relation:

$$
\rho(r)=\rho_{j}\left(1+\frac{\gamma-1}{2} M^{2} \frac{u(r)}{u_{j}}\left(1-\frac{u(r)}{u_{j}}\right)\right)^{-1}
$$

The azimuthal and radial velocities are initially null, and the pressure is equal to $p_{0}$. In order to seed the laminar-turbulent transition of the jet flow, vortex rings are added to the flow field in the shear layer at $x=r=r_{0}$, at each time step of the computation [34]. The amplitude of the perturbations is equal to $\alpha=0.007$ and the half-width of the Gaussian profile that defines the vortices is equal to $\Delta_{0}=0.045 r_{0}$. The small disturbances are divergence-free to minimize the production of spurious acoustic waves.

\subsection{Numerical set-up}

Two simulations are carried out using Cartesian grids with and without a non-conforming interface. The computational domain extends from $x=0$ up to $x=48 r_{0}$ in the flow direction and from 0 up to $20 r_{0}$ in the $y$ and $z$ directions.

The spatial discretization in the grid without a non-conforming interface is presented in Fig. 20. In the $x$ direction, for $0 \leq x \leq 25 r_{0}$, the axial mesh spacing is constant with $\Delta x=0.1 r_{0}$, and then increases with a rate of $0.4 \%$ up to $x=35 r_{0}$, and with a rate of $8 \%$ for $x>35 r_{0}$. In the $y$ and $z$ directions, the mesh is finer than in the $x$ direction in order to resolve the shear layers. The grid spacing does not vary for $y, z \leq r_{0}$, with $\Delta y=\Delta z=r_{0} / 30$. For $y, z>r_{0}$, a stretching ratio of $2 \%$ is applied up to $r=20 r_{0}$.

The non-conforming mesh is built from the conforming mesh. Figure 21 provides a simplified representation of the two meshes in the $x-y$ plane, with the non-conforming interface indicated by a bold line in Fig. 21(b). In the jet flow region, for $x \leq 14 r_{0}$, the two meshes are identical. 
(a)

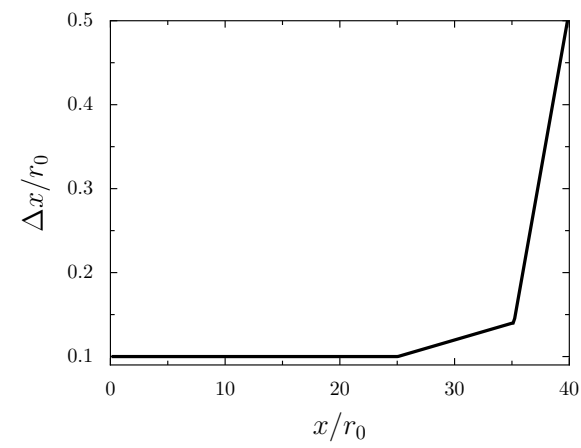

(b)

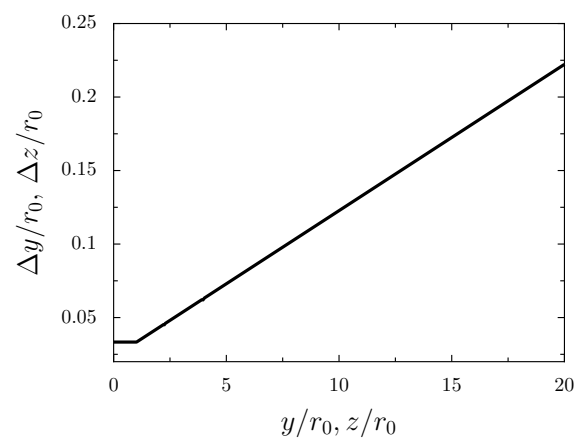

Figure 20. Representation of the spatial discretizations in the conforming grid: (a) axial discretization and (b) discretization in the $y$ and $z$ directions.

Downstream of the end of the jet potential core expected to be around $x_{p} \simeq 10 r_{0}$ according to reference [35], a non-conforming interface is defined at $x=14 r_{0}$, as shown in Fig. 21(b). The location of the interface is chosen downstream of the jet sound source region, which is found for $x \leq x_{p}$. For $x>14 r_{0}$, the very fine mesh spacings used in the $y$ and $z$ directions at the jet inlet to discretize the jet shear layers are not necessary due to the jet spreading. Therefore, downstream of the interface, the grid spacings $\Delta y$ and $\Delta z$ in the non-conforming mesh are twice as coarse as in the conforming grid. Thus, the number of mesh points in the non-conforming grid, equal to 42 million points, is reduced by $44 \%$ compared to the conforming grid.

The conforming grid used in the present work is finer than the one used in the reference [33] by factors of 1.3 and 2 in the axial and radial directions respectively. In addition, since the resolutions of the two grids used in this study differ for $x>14 r_{0}$, small differences between the results from the two simulations are expected, as demonstrated by the grid sensitivity of turbulent jet flows presented in reference [36].

(a)

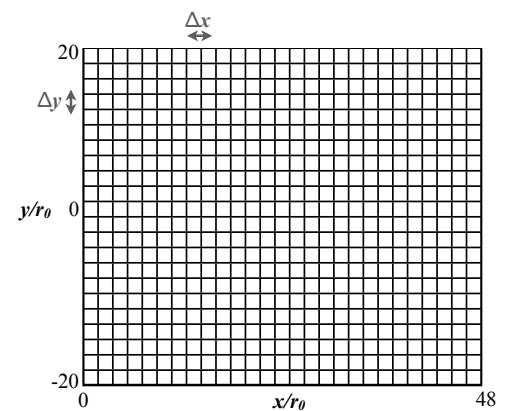

(b)

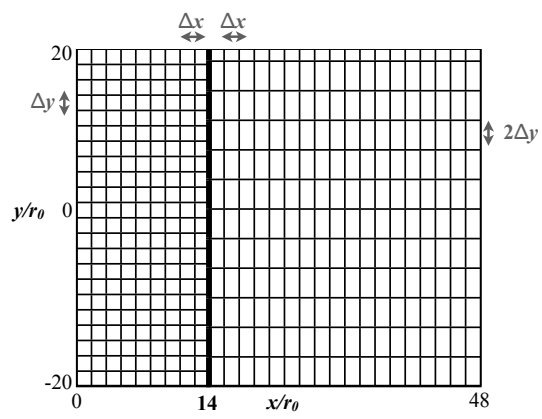

Figure 21. Sketch of (a) the conforming grid and (b) the non-conforming grid in the $x-y$ plane.

In each computation, the jet flow is simulated over a time period $T=2 \times 10^{5} \Delta t=2000 r_{0} / c_{0}$. The flow initialization lasts over $600 r_{0} / c_{0}$. The velocity and pressure fields are then recorded during a sampling period $T_{s}=1400 r_{0} / c_{0}$, leading to a minimum Strouhal number $\mathrm{St}_{\min }=D /\left(T_{s} u_{j}\right)$ of $1.6 \times 10^{-3}$. In order to study the development of the jet flow, the velocity field is recorded along the jet axis and at $r=r_{0}$. In order to examine the acoustic sound radiated in the near-field region, pressure spectra at $r=8 r_{0}$ are computed by averaging over 8 points equally distributed on circles 
centered on the jet axis. The data are sampled every 10 time steps in order to compute spectra up to a maximum Strouhal number $\mathrm{St}_{\max }=f D / u_{j}=5.55$, and the spectra are evaluated from overlapping samples of duration $93.3 r_{0} / c_{0}$.

At the non-conforming interface, the flux reconstruction for plane interfaces presented in section 3.1 is applied. The RBF interpolations are performed using $n_{v}=8$ points and seconddegree polynomial functions. The choice of the values of $n_{v}$ and $\operatorname{deg}(\mathrm{P})$ is motivated by the fact that it provided accurate results for an acoustic pulse propagation and for a vortex convection on non-conforming Cartesian grids in two-dimensional problems (see sections 5 and 6.1).

\subsection{Results}

Snapshots of the vorticity magnitude and the fluctuating pressure obtained in the two simulations are presented in Fig. 22. The non-conforming interface at $x=14 r_{0}$ is indicated by a vertical line in Fig. 22(b). In the two simulations, the jet mixing layers are found to develop from $x=r_{0}$ and to interact around $x=12 r_{0}$. Farther downstream, in Fig. 22(b), vortical structures cross the nonconforming interface and display lower levels than those located upstream for $x<14 r_{0}$. These levels are also lower than those obtained for $x>14 r_{0}$ in Fig. 22(a). This is most likely due to the mesh resolution that is twice as coarse in the $y$ direction for $x>14 r_{0}$ in the non-conforming grid [36]. In the pressure field, acoustic waves propagate from the jet with an angle of about $30^{\circ}$ relative to the $x$-axis. In Fig. 22(b), no discontinuity of the pressure waves radiated from the jet and no spurious reflection are visible in the vicinity of the non-conforming interface.

(a)

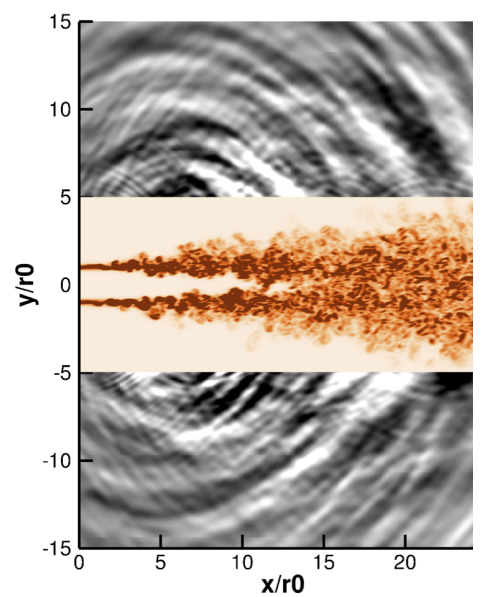

(b)

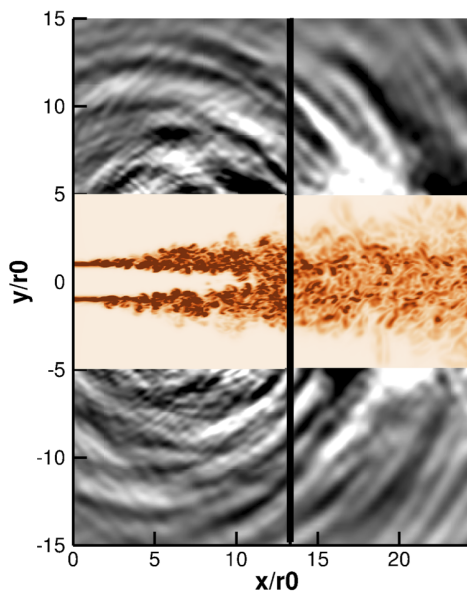

Figure 22. Snapshots in the $x-y$ plane of the vorticity modulus in the flow and of the fluctuating pressure outside obtained from the simulations using (a) a conforming grid and (b) a non-conforming grid. The color scale is from 0 to $2.5 u_{j} / r_{0}$ for the vorticity and the grey scale is from -70 to $70 \mathrm{~Pa}$ for the pressure.

More quantitative results are shown in Fig. 23, where the mean axial velocity profiles obtained in the two simulations are given along the jet axis and at $r=r_{0}$. In Fig. 23(a), the two profiles along the jet axis are superimposed for $x \leq 20 r_{0}$. They indicate that in both jets, the jet potential core ends at $x_{p}=12 r_{0}$. For $x>20 r_{0}$, slightly lower velocity values are found using the non-conforming grid, with differences that do not exceed $0.025 u_{j}$. In Fig. 23(b), the velocity profiles at $r=r_{0}$ are also 
superimposed upstream of the grid interface, whereas velocity is lower by $0.02 u_{j}$ for $x>14 r_{0}$ in the simulation using a non-conforming grid.

(a)

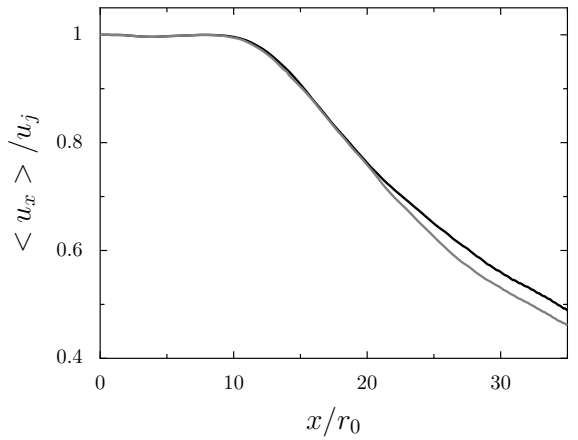

(b)

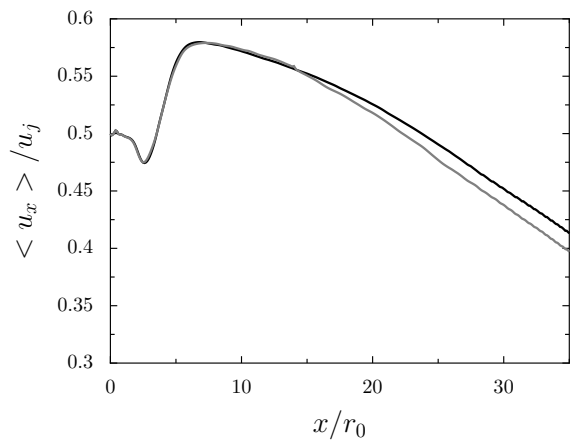

Figure 23. Representation of the mean axial velocity (a) along the jet centerline and (b) at $r=r_{0}$ : simulations with — a conforming grid and a non-conforming grid.

The rms axial and radial velocities $<u_{x}^{\prime} u_{x}^{\prime}>^{1 / 2} / u_{j}$ and $<u_{r}^{\prime} u_{r}^{\prime}>^{1 / 2} / u_{j}$ calculated at $r=r_{0}$ are represented in Fig. 24, where the prime stands for the fluctuating quantity and $\langle\cdot\rangle$ for time average. In Fig. 24(a) and 24(b), in the same way as for the mean profiles of Fig. 23(b), the profiles from the two simulations are identical for $x<14 r_{0}$, with the same peaks of turbulence. For $x \geq 14 r_{0}$, small differences of less than $1 \%$ appear between the two computations. In particular, using a non-conforming grid, the rms profiles slightly decrease at the non-conforming interface $x=14 r_{0}$ and present a small hump downstream for $17 r_{0} \leq x \leq 27 r_{0}$. Despite this, for $x \geq 27 r_{0}$, very similar turbulent levels are found in the two simulations. The small differences reported between the two simulations in Fig. 23 and 24 are likely due to the resolution of the non-conforming grid that is coarser for $x \geq 14 r_{0}$ [36].

(a)

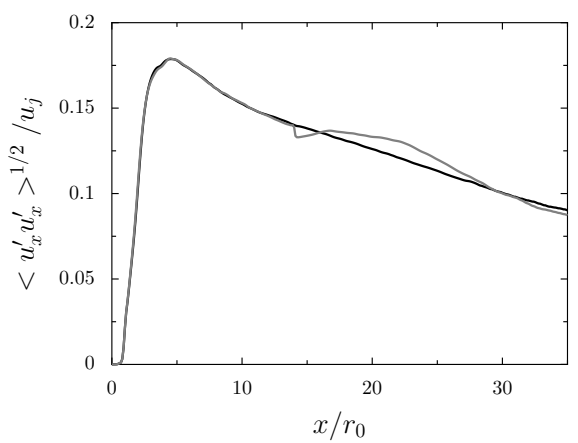

(b)

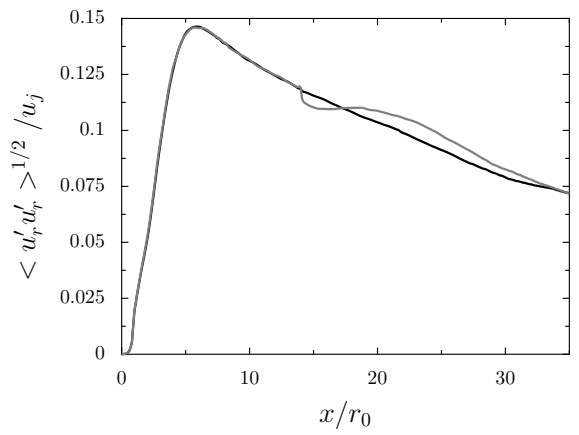

Figure 24. Representation of the (a) axial and (b) radial rms velocity profiles at $r=r_{0}$ : simulations with - a conforming grid and - a non-conforming grid.

In order to investigate the effects of the presence of the non-conforming interface on the jet flow features, axial velocity spectra are computed at three locations along the jet axis, upstream of the non-conforming interface at $x=13.4 r_{0}$, and downstream of the interface at $x=14.7 r_{0}$ and at $x=20 r_{0}$. The spectra are represented in Fig. 25 as a function of the Strouhal number. At $x=13.4 r_{0}$, the spectra exhibit similar shapes and levels, which suggests that the jet flow components are not affected by the close proximity of the non-conforming interface. Downstream 
(a)

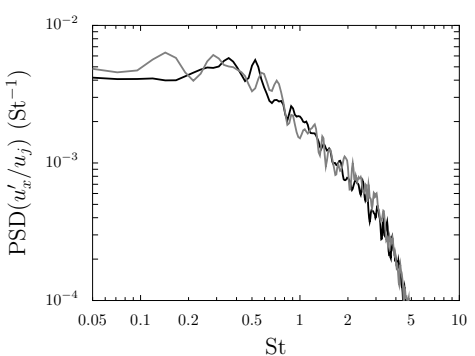

(b)

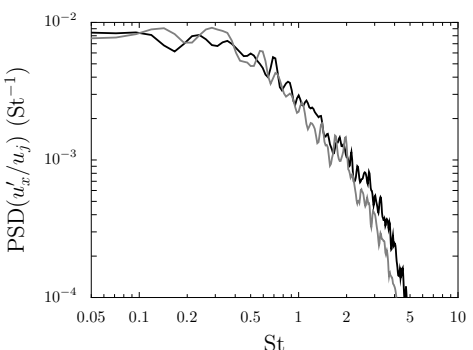

(c)

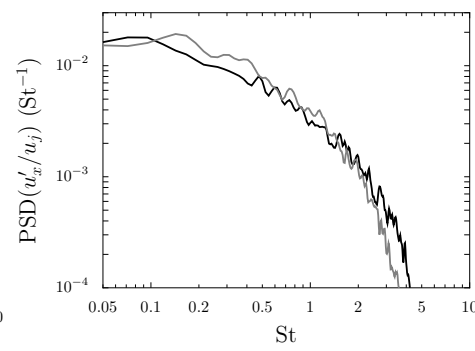

Figure 25. Representation of the axial velocity spectra on the jet axis (a) upstream of the grid interface at $x=13.4 r_{0}$, and downstream at (b) $x=14.7 r_{0}$ and (c) $x=20 r_{0}:-$ conforming grid and - nonconforming grid.

(a)

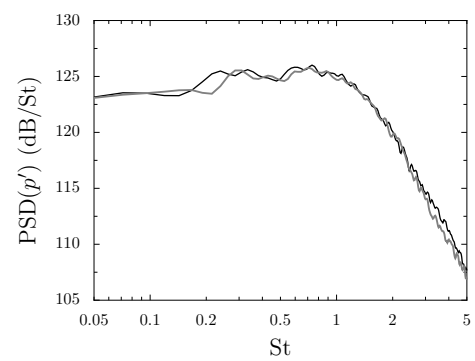

(b)

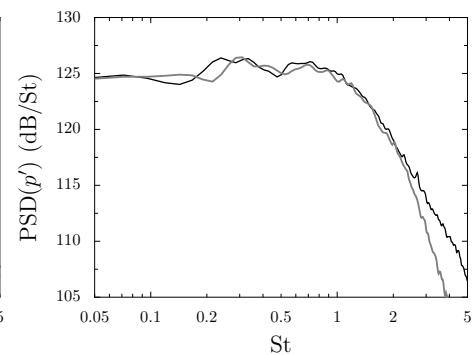

(c)

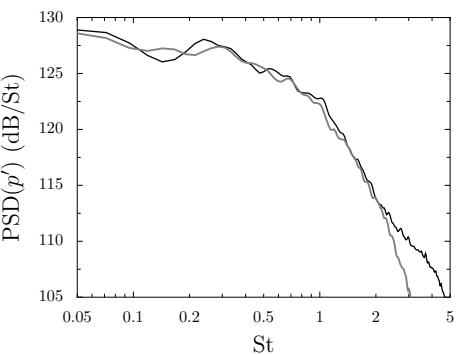

Figure 26. Representation of the pressure spectra at $r=8 r_{0}$ (a) upstream of the grid interface at $x=13.4 r_{0}$, and downstream at (b) $x=14.7 r_{0}$ and (c) $x=20 r_{0}:-$ conforming grid and — non-conforming grid.

Finally, to examine the acoustic results in the near-field region, pressure spectra at $r=8 r_{0}$ are plotted in Fig. 26 for the axial positions $x=13.4 r_{0}, x=14.7 r_{0}$ and $x=20 r_{0}$. The spectra at the three locations display broadband shapes, which is typical of subsonic turbulent jet noise [37]. Upstream of the grid interface, at $x=13.4 r_{0}$, the spectra from the two simulations are very similar, which is expected since the two meshes are identical for $x \leq 14 r_{0}$. Downstream of the interface, at $x=14.7 r_{0}$ and $x=20 r_{0}$, the acoustic spectra predicted by the two simulations are in good agreement for Strouhal numbers $\mathrm{St}<2$. For $\mathrm{St} \geq 2$, the spectrum obtained from the non-conforming grid collapses more rapidly. This drop is due to the lower mesh cut-off Strouhal number in that case. Indeed, at $r=8 r_{0}$, considering that the spatial schemes resolve wavelengths discretized by at least 5 points [14], the cut-off Strouhal number is of $\mathrm{St}_{\text {cut-off }}=2$ for the non-conforming grid and of $\mathrm{St}_{\text {cut-off }}=4$ for the conforming grid. 
These results demonstrate that the present non-conforming grid methodology can be used in order to reduce the size of the mesh and thus the computational cost, without appreciably biasing the jet development and the noise field radiated by the jet.

\section{CONCLUSION}

In this study, a flux reconstruction technique is presented in order to perform aeroacoustic computations using high-order finite-volume spatial schemes on structured meshes including nonconforming grid interfaces. The spatial discretization is carried out using a sixth-order implicit scheme in combination with a sixth-order implicit selective filter. The flux reconstruction can be applied to plane or curved non-conforming interfaces. It is performed using non-centered formulations for the spatial scheme and the selective filter at the non-conforming interface. These formulations require the definition of ghost cells and ghost interfaces. The flow variables in the ghost cells and at the interfaces are computed using meshless interpolations with radial basis functions. For computational efficiency, all the interpolation coefficients are computed once in the beginning of the simulation and then stored in memory. The properties of the RBF interpolations in the wavenumber space are studied. The accuracy of the flux reconstruction is evaluated for an acoustic pulse introduced in the vicinity of a non-conforming interface using 2-D Cartesian grids. RBF interpolations using $n_{v}=8$ points in conjunction with a 2 nd-degree polynomial function are found to be sufficient to obtain accurate results. The performance of the flux reconstruction is then examined for the convection of a vortex using 2-D Cartesian and wavy grids. The results on Cartesian grids highlight the benefits of using RBF interpolations, instead of a low-order flux reconstruction, in order to reduce the spurious pressure waves produced at the block interface. The results obtained with different spatial resolutions also show that the non-conforming grids must be designed such that the flow field is well discretized by the mesh before and after the grid interface. The results of the computations performed on wavy grids demonstrate the advantages of using the flux reconstruction for curved interfaces. More precisely, the flux reconstruction technique designed for curved interfaces produces lower spurious noise level than those obtained using the reconstruction for plane interfaces. It seems also recommended to use low curvature non-conforming interfaces. Finally, the application of the flux reconstruction technique to 3-D flows is illustrated for a turbulent round jet flow at a diameter-based Reynolds number of $4 \times 10^{5}$. Simulations are performed with and without a non-conforming grid interface downstream of the jet potential core. The jet development is only slightly affected by the presence of the non-conforming grids, and the acoustic spectra in the near-field region are very similar.

\section{ACKNOWLEDGMENTS}

This study, financially supported by Airbus, was performed at CERFACS in the context of the $\mathrm{PhD}$ thesis of the first author. The authors gratefully acknowledge Dr. Marc Montagnac for his crucial help in the development of the numerical code used in this work. The post-processing of the numerical results is carried out using Antares (release 1.11.0, https://www.cerfacs.fr/antares) [38]. 


\section{APPENDIX}

\section{A. CALCULATION OF RBF INTERPOLATION COEFFICIENTS}

\section{A.1. Interpolation of the flow variables in the ghost cells}

As presented in section 3.1.3, in order to reconstruct the flow variables in the ghost cells, RBF interpolations are performed. A RBF interpolation $u_{\mathrm{RBF}}$ of the variable $u$ at point $\boldsymbol{x}$ is defined as a linear combination of Wendland's radial basis functions $\Phi$ and a polynomial term:

$$
u_{\mathrm{RBF}}(\boldsymbol{x})=\sum_{j=1}^{n_{v}} \xi_{j} \Phi\left(\boldsymbol{x}, \boldsymbol{x}_{j}\right)+\sum_{q=1}^{m} \zeta_{q} P_{q}(\boldsymbol{x})
$$

where $\xi_{j}$ and $\zeta_{q}$ are the unknown interpolation coefficients. In this study, $\mathcal{C}^{2}$ Wendland's basis functions $[9,28]$ with compact support are used:

$$
\Phi\left(\boldsymbol{x}, \boldsymbol{x}_{j}\right)=\Phi\left(r_{j}\right)=\left(1-\frac{r_{j}}{R_{v}}\right)_{+}^{4}\left(4 \frac{r_{j}}{R_{v}}+1\right) \quad 1 \leq j \leq n_{v}
$$

where $r_{j}$ is the Euclidian distance between the points $\boldsymbol{x}$ and $\boldsymbol{x}_{j},\left(1-\frac{r_{j}}{R}\right)_{+}$is defined by:

$$
\left(1-\frac{r_{j}}{R_{v}}\right)_{+}= \begin{cases}\left(1-\frac{r_{j}}{R_{v}}\right) & \text { if } 0 \leq r_{j} \leq R_{v} \\ 0 & \text { if } r_{j}>R_{v}\end{cases}
$$

and $R_{v}$ is the radius of the circle of center $x$ defined such that $R_{v}=A r_{\text {min }}$, with $r_{\text {min }}=\min \left(r_{j}\right)_{\left(j=1, . ., n_{v}\right)}$ and $A$ is a value chosen such that $n_{v}$ cells are contained inside the circle. A representation of the ghost cell and the $n_{v}$ cells is provided in Fig. 27.

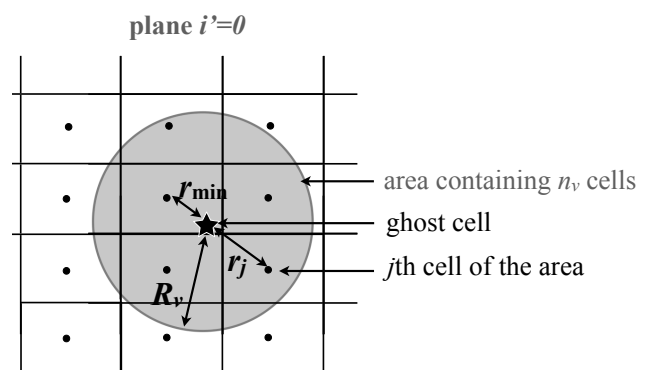

Figure 27. Representation of the cloud of $n_{v}$ cells used for the interpolation of the flow variables in the ghost cell. The center of the ghost cell is indicated by a star.

The values of $\xi_{j}$ and $\zeta_{q}$ are determined so that the approximation $u_{\mathrm{RBF}}(\boldsymbol{x})$ is exact for all the $n_{v}$ points. Therefore, the interpolation formulation (40) satisfies the following relations:

$$
u_{\mathrm{RBF}}\left(\boldsymbol{x}_{k}\right)=u_{k}=\sum_{j=1}^{n_{v}} \xi_{j} \Phi\left(\boldsymbol{x}_{k}, \boldsymbol{x}_{j}\right)+\sum_{q=1}^{m} \zeta_{q} P_{q}\left(\boldsymbol{x}_{k}\right) \text { for } 1 \leq k \leq n_{v}
$$

where $\left(u_{k}\right)_{k=1, . ., n_{v}}$ are the values of $u$ known in the $n_{v}$ cells considered for the interpolation. In order to ensure that approximation (40) has a unique solution [18,29], the following orthogonality 
691

constraints are imposed:

$$
\sum_{j=1}^{n_{v}} P_{q}\left(\boldsymbol{x}_{j}\right) \xi_{j}=0 \text { for } 1 \leq q \leq m
$$

692

693 694

where $\boldsymbol{\xi}=\left(\xi_{1}, \ldots, \xi_{n_{v}}\right)^{T}$ and $\boldsymbol{\zeta}=\left(\zeta_{1}, \ldots, \zeta_{m}\right)^{T}$ are the vectors of the interpolation coefficients to be determined, $\mathbf{u}^{\text {set }}=\left(u_{1}, \ldots, u_{n_{v}}\right)^{T}$, and $\boldsymbol{\Phi} \in \mathbb{R}^{n_{v} \times n_{v}}$ and $\mathbf{P} \in \mathbb{R}^{n_{v} \times m}$ are the matrices defined by:

$$
\begin{aligned}
& \boldsymbol{\Phi}_{k j}=\Phi\left(\boldsymbol{x}_{k}, \boldsymbol{x}_{j}\right) \text { for } 1 \leq k, j \leq n_{v} \\
& \mathbf{P}_{k q}=P_{q}\left(\boldsymbol{x}_{k}\right) \text { for } 1 \leq k \leq n_{v} \text { and } 1 \leq q \leq m
\end{aligned}
$$

The solution of the system (45) writes:

$$
\left(\begin{array}{l}
\boldsymbol{\xi} \\
\boldsymbol{\zeta}
\end{array}\right)=\mathbf{M}^{-1}\left(\begin{array}{c}
\mathbf{u}^{\text {set }} \\
\mathbf{0}
\end{array}\right)=\left(\begin{array}{c}
\sum_{k=1}^{n_{v}} m_{1, k} u_{k} \\
. . \\
. . \\
\sum_{k=1}^{n_{v}} m_{n_{v}+m, k} u_{k}
\end{array}\right)
$$

where $m_{i, j}$ are the coefficients of the matrix $\mathbf{M}^{-1}$. Introducing Eq. (47) in Eq. (40), the RBF interpolation applied to a ghost cell of center $x_{\text {ghost }}$ is given by the relation:

$$
u_{\mathrm{RBF}}\left(\boldsymbol{x}_{\text {ghost }}\right)=\sum_{k=1}^{n_{v}} \Psi_{k}\left(\boldsymbol{x}_{\text {ghost }}\right) u_{k}
$$

where the coefficients $\Psi_{k}\left(x_{\text {ghost }}\right)$ are defined as:

$$
\Psi_{k}\left(\boldsymbol{x}_{\text {ghost }}\right)=\sum_{j=1}^{n_{v}} m_{j, k} \Phi\left(\boldsymbol{x}_{\text {ghost }}, \boldsymbol{x}_{j}\right)+\sum_{q=1}^{m} m_{n_{v}+q, k} P_{q}\left(\boldsymbol{x}_{\text {ghost }}\right)
$$

Note that the $n_{v}$ coefficients $\Psi_{k}\left(x_{\text {ghost }}\right)$ are independent of the flow variables. Therefore, they are computed and stored in memory at the beginning of the simulation. The interpolation is therefore performed at a low CPU cost since the value of $u_{\mathrm{RBF}}$ is simply obtained from the sum (48).

\section{A.2. Interpolation of the flow variables at the non-conforming grid interface}

A.2.1. Plane interfaces As described in section 3.1.3, the interpolation formulation at the block interface writes: 


$$
\begin{aligned}
\tilde{u}_{L^{\prime}} & =\frac{1}{\left|I_{L}^{\prime}\right|} \int_{I_{L}^{\prime}} u_{\mathrm{RBF}}(\boldsymbol{x}) \mathrm{d} \boldsymbol{x} \\
& =\sum_{j^{\prime}=1}^{n_{v}} \widetilde{\xi}_{j^{\prime}}\left(\frac{1}{\left|I_{L}^{\prime}\right|} \int_{I_{L}^{\prime}} \Phi\left(\boldsymbol{x}, \boldsymbol{x}_{j^{\prime}}\right) \mathrm{d} \boldsymbol{x}\right)+\sum_{q=1}^{m} \widetilde{\zeta}_{q}\left(\frac{1}{\left|I_{L}^{\prime}\right|} \int_{I_{L}^{\prime}} P_{q}(\boldsymbol{x}) \mathrm{d} \boldsymbol{x}\right)
\end{aligned}
$$

711

712

713

714

As in section A.1, the RBF interpolation (50) can be reformulated as:

$$
\tilde{u}_{L^{\prime}}=\sum_{k=1}^{n_{v}} \widetilde{\Psi}_{k} u_{k}
$$

715 where the coefficients $\widetilde{\Psi}_{k}$ are defined as:

$$
\widetilde{\Psi}_{k}=\sum_{j^{\prime}=1}^{n_{v}} \frac{1}{\left|I_{L}^{\prime}\right|} \int_{I_{L}^{\prime}} m_{j^{\prime}, k}^{\prime} \Phi\left(\boldsymbol{x}, \boldsymbol{x}_{j^{\prime}}\right) \mathrm{d} \boldsymbol{x}+\sum_{q=1}^{m} m_{n_{v}+q, k}^{\prime} \frac{1}{\left|I_{L}^{\prime}\right|} \int_{I_{L}^{\prime}} P_{q}(\boldsymbol{x}) \mathrm{d} \boldsymbol{x}
$$

716 where $m_{i, j}^{\prime}$ are the coefficients of the inverse of the matrix of the system (52). In practice, the values 717 of $\widetilde{\Psi}_{k}$ are computed only once and then stored in memory at the beginning of the simulation. 


$$
\mathbf{U}_{i^{\prime}=0, j}=\frac{S_{\mathrm{AM}}}{S_{\mathrm{AB}}} \mathbf{U}_{i^{\prime}=0, j^{\prime}}+\frac{S_{\mathrm{BM}}}{S_{\mathrm{AB}}} \mathbf{U}_{i^{\prime}=0, j^{\prime}+1}
$$

where $S_{k}$ is the surface of interface $I_{k}$. Similarly, the value of $\mathbf{U}$ in the second ghost cell in Fig. 5(a) is calculated as the weighted sum of $\mathbf{U}$ in the cells $\left(i^{\prime}=1, j^{\prime}\right)$ and $\left(i^{\prime}=1, j^{\prime}+1\right)$.

A.3.2. Interpolation at the grid interface In order to compute the convective flux at the nonconforming interface, the value of the vector $\widetilde{\mathbf{U}}$ at the ghost interface $I_{L}^{\prime}$ in block R is computed 


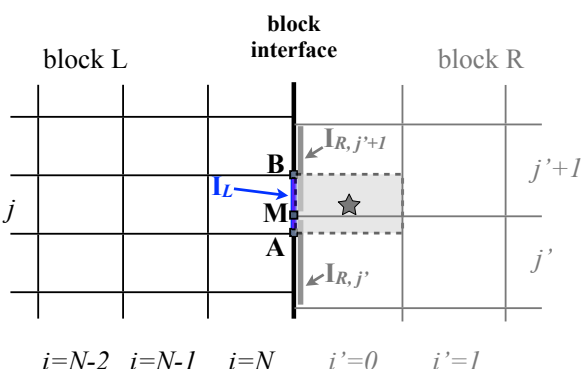

Figure 28. Representation of a non-conforming grid where the interface $I_{L}$ is divided in two interfaces $I_{\mathrm{AM}}$ and $I_{\mathrm{MB}}$. The flow variables in the ghost cell indicated in grey are computed as a weighted sum of the flow variables in cells $\left(i^{\prime}=0, j^{\prime}\right)$ and $\left(i^{\prime}=0, j^{\prime}+1\right)$.

as the weighted sum:

$$
\widetilde{\mathbf{U}}_{L}^{\prime}=\frac{S_{\mathrm{AM}}}{S_{\mathrm{AB}}} \widetilde{\mathbf{U}}_{R, j^{\prime}}+\frac{S_{\mathrm{BM}}}{S_{\mathrm{AB}}} \widetilde{\mathbf{U}}_{R, j^{\prime}+1}
$$

where the values of $\widetilde{\mathbf{U}}$ at the interfaces $I_{R, j^{\prime}}$ and $I_{R, j^{\prime}+1}$ are computed from the upwind scheme (14). The value of $\widetilde{\mathbf{U}}$ at the interface $I_{L}$ is calculated from the upwind scheme (13). Finally, the convective flux at the block interface is determined from the values of $\widetilde{\mathbf{U}}_{L}$ and $\widetilde{\mathbf{U}}_{L}^{\prime}$ by resolving a Riemann flux problem [25]. 9(1-2):123-143, 2010.

2. C Bogey and C Bailly. A family of low dispersive and low dissipative explicit schemes for flow and noise computations. Journal of Computational Physics, 194(1):194-214, 2004.

3. T Colonius and SK Lele. Computational aeroacoustics: progress on nonlinear problems of sound generation. Progress in Aerospace Sciences, 40(6):345-416, 2004.

4. C Bogey, O Marsden, and C Bailly. Large-eddy simulation of the flow and acoustic fields of a Reynolds number $10^{5}$ subsonic jet with tripped exit boundary layers. Physics of Fluids, 23:035104, 2011.

5. C Bogey and C Bailly. Influence of nozzle-exit boundary-layer conditions on the flow and acoustic fields of initially laminar jets. Journal of Fluid Mechanics, 663:507-538, 2010.

6. GA Brès, P Jordan, T Colonius, M Le Rallic, V Jaunet, and SK Lele. Large eddy simulation of a Mach 0.9 turbulent jets. Proceedings of the Summer Program, pages 221-230, 2014.

7. D Desvigne, O Mardsen, C Bogey, and C Bailly. Development of noncentered wavenumber-based optimized interpolation schemes with amplification control for overlapping grids. SIAM Journal on Scientific Computing, 32(4):2074-2098, 2010.

8. J Huber, G Drochon, A Pintado-Peno, F Cléro, and G Bodard. Large-Scale Jet Noise Testing, Reduction and Methods validation "EXEJET": 1. Project Overview and Focus on Installation. 20th AIAA/CEAS Aeroacoustics Conference, paper AIAA-2014-3032, 2014.

9. WJ Costin and CB Allen. Numerical study of radial basis function interpolation for data transfer across discontinuous mesh interfaces. International Journal for Numerical Methods in Fluids, 72(10):1076-1095, 2013.

10. CKW Tam and JC Webb. Dispersion-relation-preserving finite difference schemes for computational acoustics. Journal of Computational Physics, 107:262-281, 1993.

11. SK Lele. Compact finite difference schemes with spectral-like resolution. Journal of Computational Physics, 103:16-42, 1992.

12. F Fosso Pouangué, H Deniau, F Sicot, and P Sagaut. Curvilinear finite volume scheme using high order compact interpolation. Journal of Computational Physics, 229(13):5090-5122, 2010.

13. MR Visbal and DV Gaitonde. On the use of higher-order finite-difference schemes on curvilinear and deforming meshes. Journal of Computational Physics, 181:155-185, 2002. 
14. A Fosso Pouangué, M Sanjosé, S Moreau, G Daviller, and H Deniau. Subsonic jet noise simulations using both structured and unstructured grids. AIAA Journal, 53(1):55-69, 2014.

15. R Biolchini, C Bailly, J-F Boussuge, and R Fernando. Numerical study on the relation between hydrodynamic fluctuations and noise in hot jets at high Reynolds numbers. In 22nd AIAA/CEAS Aeroacoustics Conference, paper AIAA-2016-3048, 2016.

16. P Grenson and H Deniau. Large-eddy simulation of an impinging heated jet for a small nozzle-to-plate distance and high Reynolds number. International Journal of Heat and Fluid Flow, 68:348-363, 2017.

17. CP Arroyo, G Daviller, G Puigt, C Airiau, and S Moreau. Identification of temporal and spatial signatures of broadband shock-associated noise. Shock Waves, pages 1-18, 2018.

18. JG Wang and GR Liu. A point interpolation meshless method based on radial basis functions. International Journal for Numerical Methods in Engineering, 54(11):1623-1648, 2002.

19. S Le Bras, H Deniau, and C Bogey. A flux reconstruction technique for non-conforming grid interfaces in aeroacoustic simulations. In 22nd AIAA/CEAS Aeroacoustics Conference, paper AIAA-2016-2972, 2016.

20. L Cambier, S Heib, and S Plot. The Onera elsA CFD Software: Input from Research and Feedback from Industry. Mechanics \& Industry, 14(03):159-174, 2013.

21. M Lazareff, AM Vuillot, and L Cambier. Manuel théorique du logiciel elsA, 2010.

22. D Fauconnier, C Bogey, and E Dick. On the performance of relaxation filtering for large-eddy simulation. Journal of Turbulence, 14(1):22-49, 2013.

23. F Kremer and C Bogey. Large-eddy simulation of turbulent channel flow using relaxation filtering: Resolution requirement and Reynolds number effects. Computers \& Fluids, 116:17-28, 2015.

24. S Le Bras, H Deniau, C Bogey, and G Daviller. Development of compressible large-eddy simulations combining high-order schemes and wall modeling. AIAA Journal, pages 1-12, 2017.

25. EF Toro, M Spruce, and W Speares. Restoration of the contact surface in the HLL-Riemann solvers. Shock waves, 4(1):25-34, 1994

26. A Fosso Pouangué. Schémas Volumes Finis Précis: application à l'aéroacoustique numérique de jets subsoniques. PhD thesis, Université Pierre et Marie Curie, 2011.

27. DV Gaitonde and MR Visbal. High-order schemes for Navier-Stokes equations: algorithm and implementation into fdl3di. Technical report, DTIC Document, 1998.

28. H Wendland. Scattered data approximation, volume 17. Cambridge University Press, 2004.

29. MA Golberg, CS Chen, and H Bowman. Some recent results and proposals for the use of radial basis functions in the BEM. Engineering Analysis with Boundary Elements, 23(4):285-296, 1999.

30. JC Carr, RK Beatson, JB Cherrie, TJ Mitchell, WR Fright, BC McCallum, and TR Evans. Reconstruction and representation of 3D objects with radial basis functions. In Proceedings of the 28th annual conference on Computer graphics and interactive techniques, pages 67-76. ACM, 2001.

31. CKW Tam and JC Webb. Dispersion-relation-preserving finite difference schemes for computational acoustics. Journal of Computational Physics, 107(2):262-281, 1993.

32. G Fillola, MC Le Pape, and M Montagnac. Numerical simulations around wing control surfaces. 24th International Council of Aeronautical Sciences, paper ICAS-2004-2.3.2, 2004.

33. C Bogey and C Bailly. Computation of a high Reynolds number jet and its radiated noise using large eddy simulation based on explicit filtering. Computers \& Fluids, 35(10):1344-1358, 2006.

34. C Bogey, C Bailly, and D Juvé. Noise investigation of a high subsonic, moderate Reynolds number jet using a compressible large eddy simulations. Theoretical and Computational Fluid Dynamics, 16(4):273-297, 2003.

35. JC Lau, PJ Morris, and MJ Fisher. Measurements in subsonic and supersonic free jets using a laser velocimeter. Journal of Fluid Mechanics, 93(01):1-27, 1979.

36. C Bogey. Grid sensitivity of flow field and noise of high-Reynolds-number jets computed by large-eddy simulations. International Journal of Aeroacoustics, 17(4-5):399-424, 2018.

37. CKW Tam, K Viswanathan, KK Ahuja, and J Panda. The sources of jet noise: experimental evidence. Journal of Fluid Mechanics, 615:253-292, 2008.

38. Antares Development Team. Antares Documentation Release 1.11.0, 2012-2018. 